\title{
ERROR BOUNDS FOR GLIMM DIFFERENCE APPROXIMATIONS FOR SCALAR CONSERVATION LAWS
}

BY

DAVID HOFF ${ }^{1}$ AND JOEL SMOLLER ${ }^{2}$

\begin{abstract}
We derive error bounds for the Glimm difference approximation to the solution of a genuinely nonlinear scalar conservation law with $\mathrm{BV}$ initial data. We show that the $L^{1}$ error is bounded by $O\left(\Delta x^{1 / 6}|\log \Delta x|\right)$ in the general case, and by $O\left(\Delta x^{1 / 2}|\log \Delta x|\right)$ for a generic class of piecewise constant data.
\end{abstract}

1. Introduction. In this paper we derive bounds for the error in the Glimm difference approximation $[\mathbf{G}]$ to the solution of a single conservation law

$$
v_{t}+f(v)_{x}=0, \quad(x, t) \in \mathbf{R} \times \mathbf{R}_{+},
$$

with initial data

$$
v(x, 0)=v_{0}(x)
$$

Here $v_{0}$ is a function of bounded variation (not necessarily small), and $f$ is a $C^{2}$ function satisfying $f^{\prime \prime}>0$ on the closed convex hull of the range of $v_{0}$. We let $u$ denote the approximate solution of (1.1) generated from Glimm's scheme and based upon an equidistributed sequence $\left\{a_{n}\right\} \subset(-1,1)$ (as explained below). We show that, given any interval $[a, b] \subseteq \mathbf{R}$ and any real numbers $\varepsilon, t>0$, there is a constant $C$, independent of the mesh parameters, such that the following error bound holds:

$$
\|u(\cdot, t)-v(\cdot, t)\|_{L^{1}[a, b]} \leq C\left[\left\|u(\cdot, 0)-v_{0}\right\|_{L^{1}[\tilde{a}, \tilde{b}]}+\Delta x^{1 / 6} \mid \log \Delta x \|\right],
$$

where $\tilde{a}=a-L t-\varepsilon, \tilde{b}=b+L t+\varepsilon$, and $L=\sup _{|v| \leq\left\|v_{0}\right\|_{\infty}}\left|f^{\prime}(v)\right|$. In addition, we show that for a generic class of piecewise constant data $v_{0}$, the error bound can be improved to $O\left(\Delta x^{1 / 2}|\log \Delta x|\right)$ when $u(\cdot, 0)$ is suitably chosen. By comparison, it is known that the error in approximations generated from monotone finite difference schemes in conservation law form is $O\left(\Delta x^{1 / 2}\right)$; see $[\mathbf{K u}]$.

In $\S 2$ we give a description of the Glimm scheme and derive the basic estimates required for the subsequent analysis. Although these estimates are well known, their derivations in the scalar case are quite simple, and we have included them in order to make our exposition self-contained. In $\S 3$ we derive an estimate for the weak truncation error associated with an approximate solution $u$. This estimate is based upon Liu's wave-tracking analysis $[\mathbf{L}]$ and requires that the equidistributed

Received by the editors June 15, 1984.

1980 Mathematics Subject Classification. Primary 35L65, 35L67, 39A10.

${ }_{1}^{1}$ Research supported in part by the NSF under Grant No. MCS-8301141.

${ }^{2}$ Research supported in part by the NSF under Grant No. MCS-8002337. 
sequence $\left\{a_{n}\right\}$ satisfy the following: There is a constant $c$ such that, given any $\theta \in[-1,1]$ and $K, N \in \mathbf{Z}_{+}$,

$$
\left|\frac{\#\left\{n \in[K+1, K+N]: a_{n} \leq \theta\right\}}{N}-\frac{1+\theta}{2}\right| \leq \frac{c \log N}{N} ;
$$

see $[\mathbf{K u ~ N}]$ for a description of such sequences. Then in $\S 4$ we combine our bound for the weak truncation error with a refined version of the stability analysis for (1.1), due to Krushkov $[\mathbf{K}]$, in order to derive the bound (1.3). Finally, in $\S 5$ we derive the improved error bound $O\left(\Delta x^{1 / 2}|\log \Delta x|\right)$ for a special class of initial data. This result is obtained by direct comparison of $u$ with $v$, and, as a consequence, $\S 5$ is independent of the rest of the paper.

\section{Preliminaries.}

A. Glimm approximate solutions. We give here a brief summary of the main properties concerning Glimm approximate solutions of (1.1), (1.2); for the general statements and more details, see $[\mathbf{G}, \mathbf{G L}, \mathbf{S}]$.

Let $\left\{a_{m}\right\}$ be a fixed equidistributed sequence in $(-1,1)$, and let $\Delta x, \Delta t$ be fixed mesh parameters which satisfy the C.F.L. condition

$$
\sup _{|u| \leq\left\|v_{0}\right\|_{\infty}}\left|f^{\prime}(u)\right| \leq \Delta x / \Delta t .
$$

The Glimm approximate solutions are solutions of a difference scheme which can be described as follows. First we define $x_{k}=k \Delta x, k \in \mathbf{Z}$, and $t_{n}=n \Delta t, n \in \mathbf{Z}$, and consider the grid in $t \geq 0$ defined by the two families of lines $x=x_{k}, k \in$ $\mathbf{Z}, t=t_{n}, n \in \mathbf{Z}_{+}$. The lines $t=t_{n}$ are partitioned into disjoint intervals $[(k-1) \Delta x,(k+1) \Delta x]$, where $k+n$ is even. In each such interval we choose a point $\theta_{k, n}=k \Delta x+a_{n} \Delta x$. We approximate the initial data by piecewise constant data $\left\{u_{k}^{0}\right\}$, where $u_{k}^{0}$ is the value of the approximation on $\left[x_{k-1}, x_{k+1}\right]$. Inductively, we solve the corresponding "Riemann problem", i.e. we solve (1.1) with data

$$
v_{0}(x)= \begin{cases}u_{k-1}^{n}, & x<k \Delta x \\ u_{k+1}^{n}, & x>k \Delta x .\end{cases}
$$

The solution of this problem is a shock wave if $u_{k-1}^{n}>u_{k+1}^{n}$ and a rarefaction wave if $u_{k+1}^{n}>u_{k-1}^{n}$ (see [S]). This defines the approximate solution $u^{n}(x, t)$ in the strip $t_{n} \leq t<t_{n+1}$. In order to obtain a piecewise constant approximation on the line $t=t_{n+1}$, we define

$$
u^{n+1}\left(x, t_{n+1}\right)=u^{n}\left(\theta_{k, n}, t_{n}\right), \quad x_{k-1} \leq x<x_{k+1} .
$$

This defines the Glimm approximate solution $u$ (which depends on the given mesh parameters $\Delta x, \Delta t)$. Notice that $u$ is an exact solution in each of the strips $t_{n} \leq$ $t<t_{n+1}$ and has jump discontinuities across each of the lines $t=t_{n}, n \geq 1$. We use the notation

$$
u_{k}^{n}=u(k \Delta x, n \Delta t), \quad(k, n) \in \mathbf{Z} \times \mathbf{Z}_{+} .
$$

We then have the following lemma.

LEMMA 2.1. (1) The "maximum principle" holds for $u$; i.e. for all $(k, n) \in$ $\mathbf{Z} \times \mathbf{Z}_{+},\left|u_{k}^{n}\right| \leq\left\|u_{0}\right\|_{\infty}$. 


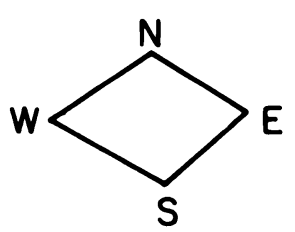

(A)

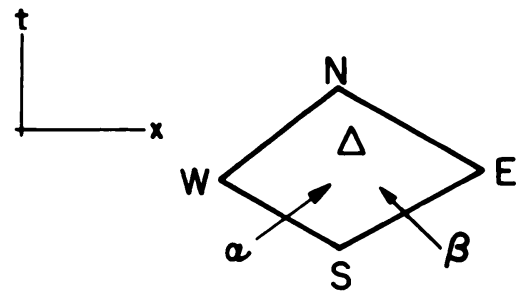

(B)

FIGURE 1

(2) The total variation is "conserved"; i.e.

$$
\sum_{k \in \mathbf{Z}}\left|u_{k+1}^{n}-u_{k-1}^{n}\right| \leq V \equiv \text { T.V. }\left(u_{0}\right) .
$$

PROOF. (1) is obvious since the solution of the Riemann problem for (1.1) with data $\left(u_{l}, u_{r}\right)$ lies in the convex hull of the points $u_{l}, u_{r}$. For $(2)$ note that, by virtue of (1), we may write

$$
u_{k}^{n+1}=\alpha_{k}^{n} u_{k-1}^{n}+\beta_{k}^{n} u_{k+1}^{n},
$$

where $\alpha_{k}^{n}+\beta_{k}^{n}=1, \alpha_{k}^{n}, \beta_{k}^{n} \geq 0$. Thus,

$$
\begin{aligned}
\sum_{k \in \mathbf{Z}} \mid & u_{k+1}^{n+1}-u_{k-1}^{n+1}\left|=\sum_{k}\right|\left(\alpha_{k+1}^{n} u_{k}^{n}+\beta_{k+1}^{n} u_{k+2}^{n}\right)-\left(\alpha_{k-1}^{n} u_{k-2}^{n}+\beta_{k-1}^{n} u_{k}^{n}\right) \mid \\
& =\sum_{k}\left|\beta_{k+1}^{n}\left(u_{k+2}^{n}-u_{k}^{n}\right)+\alpha_{k-1}^{n}\left(u_{k}^{n}-u_{k-2}^{n}\right)\right| \\
& \leq \sum_{k} \beta_{k+1}^{n}\left|u_{k+2}^{n}-u_{k}^{n}\right|+\sum_{k} \alpha_{k+1}^{n}\left|u_{k+2}^{n}-u_{k}^{n}\right| \\
& =\sum_{k}\left|u_{k+2}^{n}-u_{k}^{n}\right| .
\end{aligned}
$$

We need some notation and definitions; see $[\mathbf{G}, \mathbf{S}]$. Recall that the equidistributed points on which we do the evaluations are defined by $\left(x_{k}+a_{n} \Delta x, t_{n}\right)$. In order to estimate the total variation of an approximating solution, we cover the upper half-plane $t \geq 0$ by "diamonds", the corners of which are the equidistributed points in the mesh intervals. A mesh curve is a (nonbounded) piecewise linear curve lying on diamond boundaries going from $W$ to $N$ or $S$; see Figure 1A. $J_{0}$ denotes the (unique) mesh curve passing through the mesh points on $t=0$. If $\alpha$ is a (shock or rarefaction) wave connecting the state $u_{l}$ to the state $u_{r}$, we denote the strength of $\alpha$ by $|\alpha| \equiv\left|u_{l}-u_{r}\right|$. Finally, if $J$ is a mesh curve, we let $Q(J)=\sum|\alpha||\beta|$, where the sum is taken over all waves $\alpha, \beta$ in the approximate solution $u$ which cross $J$, and either $\alpha$ or $\beta$ is a shock wave. If $\Delta$ is the diamond depicted in Figure 1B, then for the approximate solution $u$, we define

$$
Q(\Delta)= \begin{cases}|\alpha||\beta| & \text { if } \alpha, \beta \text { enter } \Delta \\ 0 & \text { and } \alpha \text { or } \beta \text { is a shock, } \\ 0 & \text { otherwise. }\end{cases}
$$

If $R$ is a union of diamonds $\Delta_{k}$, then we define $Q(R)=\sum_{k} Q\left(\Delta_{k}\right)$. Finally,

$$
Q=Q(\{t \geq 0\})=\sum\{Q(\Delta): \Delta \subseteq[t \geq 0]\} .
$$




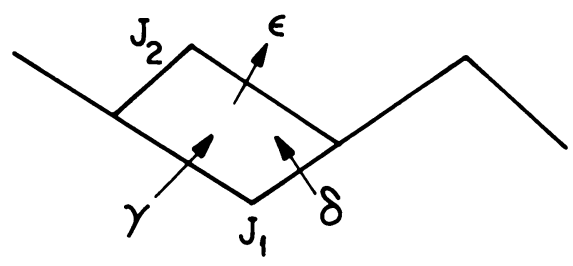

FIGURE 2

LEMMA 2.2. $Q \leq \theta V$, where $\theta=\operatorname{oscill}\left(u_{0}\right), V=\mathrm{T}$.V. $\left(u_{0}\right)$.

Proof. As usual, one shows that if $J_{2}$ is an immediate successor to $J_{1}$, then $Q\left(J_{2}\right)-Q\left(J_{1}\right) \leq-Q(\Delta)$, where $\Delta$ is the diamond between $J_{1}$ and $J_{2}$; see Figure 2. This is especially simple to prove in the scalar case since $\varepsilon=\gamma+\delta$. Thus suppose that $\alpha \notin \Delta, \alpha$ crosses $J_{1}$ and $J_{2}$, and $|\alpha \varepsilon|$ contributes to $Q\left(J_{2}\right)$. Then either (i) $\alpha<0$ or (ii) $\varepsilon<0$. In case (i), the net contribution to $Q\left(J_{2}\right)-Q\left(J_{1}\right)$ is $|\alpha \varepsilon|-|\alpha \gamma|-|\alpha \delta| \leq 0$, since $|\varepsilon| \leq|\gamma|+|\delta|$. In case (ii), one or both of $\gamma$ and $\delta$ must be negative, say $\gamma<0<\delta$. Then $0>\varepsilon=\delta+\gamma>\gamma$, and $|\alpha \varepsilon|<|\alpha \gamma|$. Thus the contribution to $Q\left(J_{2}\right)-Q\left(J_{1}\right)$ due to $\alpha$ is $|\alpha \varepsilon|-|\alpha \gamma|<0$. Finally, since $Q(\Delta)$ contributes to $Q\left(J_{1}\right)$ and not to $Q\left(J_{2}\right)$, we have $Q\left(J_{2}\right)-Q\left(J_{1}\right) \leq-Q(\Delta) \leq 0$. It follows that if $J$ is any mesh curve, and $A=\bigcup\left\{\Delta: \Delta\right.$ lies between $J$ and $\left.J_{0}\right\}$, then $Q(J) \leq Q\left(J_{0}\right)-Q(A)$, so $Q(A) \leq Q\left(J_{0}\right)$. Since this holds for all $A$, we have $Q \leq Q\left(J_{0}\right) \leq \theta V$.

Next, given a diamond $\Delta$ as above, the cancellation in $\Delta$ is defined by

$$
C(\Delta)=|\gamma|+|\delta|-|\varepsilon| \equiv|\gamma|+|\delta|-|\gamma+\delta| \text {. }
$$

We extend the domain of $C$ to $J$-curves and regions $R$ as before, and we define $C=\sum\{C(\Delta)$ : all $\Delta\}$.

\section{LEMMA 2.3. $C \leq V \equiv$ T.V. $\left(u_{0}\right)$.}

ProOF. Let $J_{n}$ be the $J$-curve passing through all mesh points on the lines $t=n \Delta t$ and $t=(n+1) \Delta t$. Let $C_{n}=\sum\left\{C(\Delta): J_{0} \leq \Delta \leq J_{n}\right\}$. If $\Delta \cap J_{0}=\emptyset=$ $\Delta \cap J_{n}$, and both $\gamma$ and $\delta$ are waves entering $\Delta$, while $\varepsilon$ is the wave leaving $\Delta$, then each of $|\varepsilon|,|\gamma|$ and $|\delta|$ is counted twice in $C_{n}$ with different signs. Hence,

$$
\begin{aligned}
C_{n} & =-\sum\left\{|\alpha|: \alpha \text { crosses } J_{n}\right\}+\sum\left\{|\alpha|: \alpha \text { crosses } J_{0}\right\} \\
& \leq \sum\left\{|\alpha|: \alpha \text { crosses } J_{0}\right\} \leq V
\end{aligned}
$$

so that $C \leq V$.

B. Wave decomposition. Let $\left\{u_{k}^{n}\right\}$ denote the states in $u$ (s€e Figure 3), and let $\left\{a_{n}\right\}$ be a given equidistributed sequence in $(-1,1)$. We partition the (time line) indices $1,2, \ldots$, into sets of equal length: $\{0 \leq n<M\},\{M \leq n<2 M\}, \ldots$, $\{(p-1) M \leq n<p M\}, \ldots$ We then have the following theorem (see $[\mathbf{L}])$.

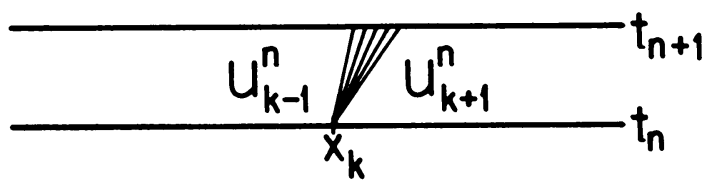

FIGURE 3 


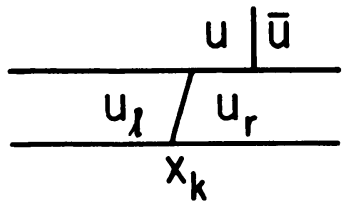

$(A)$

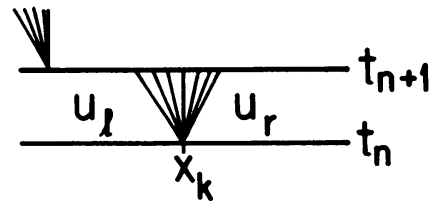

(B)

FIGURE 4

THEOREM 2.4. For $(p-1) M \leq n<p M$, one can define the following quantities:

(1) a partition of the interval $\left[u_{k \mp 1}^{n}, u_{k \pm 1}^{n}\right]: u_{k \mp 1}^{n}=y_{k, 0}^{n}<\cdots<y_{k, q_{k}^{n}}^{n}=u_{k \pm 1}^{n}$;

(2) a classification of the numbers $z_{k, j}^{n}=y_{k, j}^{n}-y_{k, j-1}^{n}$ into two disjoint sets, $\left\{\tilde{z}_{k, j}^{n}\right\}$ and $\left\{\tilde{\tilde{z}}_{k, j}^{n}\right\}$, where $z_{k, j}^{n}=\tilde{z}_{k, j}^{n}+\tilde{\tilde{z}}_{k, j}^{n}$, and one of $\tilde{z}_{k, j}^{n}, \tilde{\tilde{z}}_{k, j}^{n}$ is zero;

(3) a wave speed $\lambda_{k, j}^{n}$ for each index $(k, j)$ for which $\tilde{z}_{k, j}^{n}=0$; and

(4) an invertible map

$$
\alpha^{n}:\left\{(k, j): \tilde{z}_{k, j}^{(p-1) M} \neq 0\right\} \rightarrow\left\{(k, j): \tilde{z}_{k, j}^{n} \neq 0\right\}, \quad \alpha^{0}=\mathrm{id}
$$

such that the following properties hold:

(i) $\sum_{(k, j)}\left|\tilde{\tilde{z}}_{k, j}^{n}\right| \leq K C\left(R^{p}\right)$,

where $R^{p}=\bigcup\left\{\Delta\right.$ between $t=t_{(p-1) M}$ and $\left.t=t_{p M}\right\}$.

(ii) $\alpha^{n+1}(k, j)=\alpha^{n}(k, j)+( \pm 1, *)$.

(iii) $y_{\alpha^{n}(k, j)}^{n}=y_{k, j}^{(p-1) M}$.

(iv)

$$
\sum_{(k, j)}\left|\tilde{z}_{k, j}^{(p-1) M}\right| \max _{(p-1) M \leq n \leq p M}\left|\lambda_{\alpha^{n}(k, j)}^{n}-\lambda_{k, j}^{(p-1) M}\right| \leq K Q\left(R^{p}\right) .
$$

ProOF ( $\mathrm{LIU}[\mathbf{L}]$ ). There is no loss in generality if we take $p=1$. The proof is by induction on $n$.

We initially partition the waves so that no further partitioning occurs. Such a partition is not very difficult to achieve, but describing it is somewhat tedious. Namely, given any wave at the point $\left(x_{k_{0}}, 0\right)$, we define its "range of influence sets" by setting $A_{0}=\left\{\left(x_{k_{0}}, 0\right)\right\}$, and, given $A_{n}$, we define $A_{n+1}=\left\{\left(x_{k \pm 1}, t_{n+1}\right)\right\}$ if and only if $\left(x_{k}, t_{n}\right) \in A_{n}$, and either

(a) the shock wave at $\left(x_{k}, t_{n}\right)$ and the shock wave at $\left(x_{k \pm 1}, t_{n+1}\right)$ share a common state $\left(u_{l}\right.$ or $u_{r}$ ) (see Figure $4 \mathrm{~A}$ ), or

(b) the rarefaction wave at $\left(x_{k}, t_{n}\right)$ contains the rarefaction wave at $\left(x_{k \pm 1}, t_{n+1}\right)$; (see Figure 4B).

We further require that

$$
\left\{y_{k_{0}, j}^{0}\right\} \supseteq\left\{u_{k \pm 1}^{n}:\left(x_{k \pm 1}, t_{n}\right) \in A_{n} \text { and } u_{k \pm 1}^{n} \text { is between } u_{k_{0}-1}^{0} \text { and } u_{k_{0}+1}^{0}\right\} \text {. }
$$

(Of course, finer partitions are admissible.) We set $\tilde{z}_{k, j}^{0}=z_{k, j}^{0}$ and $\tilde{\tilde{z}}_{k, j}^{0}=0$ for all $(k, j), \alpha^{0}=$ identity, and

$$
\lambda_{k, j}^{0}= \begin{cases}f^{\prime}\left(y_{k, j}^{0}\right) & \text { if }\left(u_{k-1}^{0}, u_{k+1}^{0}\right) \text { is a rarefaction wave } \\ s\left(u_{k-1}^{0}, u_{k+1}^{0}\right) & \text { if }\left(u_{k-1}^{0}, u_{k+1}^{0}\right) \text { is a shock wave. }\end{cases}
$$

Then it is easy to see that (i)-(iv) hold (trivially or vacuously!). 


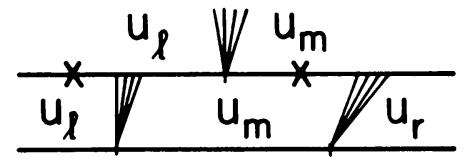

FIGURE 5

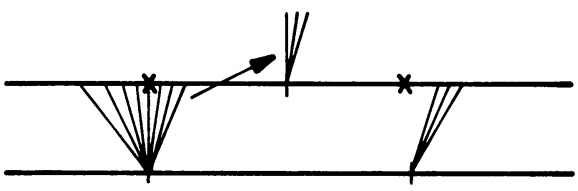

FIGURE 6

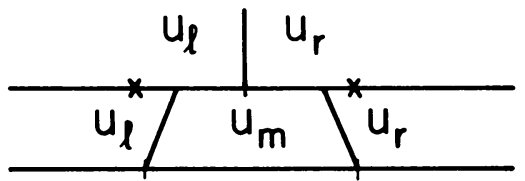

FIGURE 7

Now suppose that the partition has been constructed at time $t_{n}$. It will be sufficient to: partition the wave at $\left(x_{k}, t_{n+1}\right)$; define $\lambda_{k, j}^{n+1}$; and define $\alpha^{n+1}$ mapping the set

$$
\begin{aligned}
\left\{\left(k_{0}, j_{0}\right): \alpha^{n}\left(k_{0}, j_{0}\right)=\left(k \pm 1, j^{\prime}\right) \text { and the wave } \tilde{z}_{k \pm 1, j^{\prime}}^{n}\right. & \\
& \text { meets the line } \left.t=t_{n+1} \text { between } x_{k-1}+a_{n} \Delta x \text { and } x_{k+1}+a_{n} \Delta x\right\}
\end{aligned}
$$

onto the set $\left\{(k, j): \tilde{\tilde{z}}_{k, j}^{n+1}=0\right\}$. (In all subsequent figures waves will be centered at the points $x_{k \pm 1}, x_{k}$, and the $\times$ 's are the points $x_{k \pm 1}+a_{n} \Delta x$.) We consider several cases:

Case (a). Suppose the waves translate (cf. for example Figure 5).

Here we retain the partition of $\left[u_{l}, u_{m}\right]$, the classification, and the definition of the $\lambda$ 's. We set $\alpha^{n+1}=\alpha^{n}+( \pm 1, *)$ on the relevant domain.

Case (b). $R+R \rightarrow R^{3}$ (but different from (a); see Figure 6).

By construction, $\bar{u}$ is a partition point of $\left[u_{l}, u_{m}\right]$, so the partition of $\left[\bar{u}, u_{m}\right]$ is obtained by restriction. We retain the classification of the $\tilde{z}$ and the $\tilde{\tilde{z}}$ 's and we retain the $\lambda$ 's. Any index $\left(k_{0}, j_{0}\right)$ mapped by $\alpha^{n}$ to $\left(k-1, j^{\prime}\right)$ gets mapped to $(k, *)$ by $\alpha^{n+1}$, and thus (i)-(iv) all hold trivially.

Case (c). $S+S \rightarrow S$ (but different from (a); see Figure 7).

We have $u_{l}>u_{m}>u_{r}$, and, by induction, we have partitions of both intervals $\left[u_{r}, u_{m}\right]$ and $\left[u_{m}, u_{l}\right]$. Take the union of these partitions as the partition of $\left[u_{l}, u_{r}\right]$, retain the classifications, and define $\lambda_{k, j}^{n+1}=s\left(u_{l}, u_{r}\right)$. If $\left(k_{0}, j_{0}\right)$ is such that $\alpha^{n}\left(k_{0}, j_{0}\right)=(k \pm 1, *)$, set $\alpha^{n+1}\left(k_{0}, j_{0}\right)=(k, *)$. Then (i)-(iii) are trivial to

\footnotetext{
${ }^{3}$ We are using standard notation; namely $R$ and $S$ denote rarefaction and shock waves, respectively, and $R+R \rightarrow R$ denotes two rarefaction waves which enter a diamond and produce a rarefaction-wave. Similar meaning is given to $S+S \rightarrow S$, etc.
} 


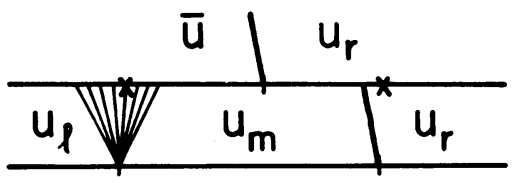

FIGURE 8

verify. For (iv) define the sets

$$
\begin{aligned}
& A=\left\{\left(k_{0}, j_{0}\right) ; \alpha^{n+1}\left(k_{0}, j_{0}\right)=(k, *)\right\}, \\
& A_{ \pm}=\left\{\left(k_{0}, j_{0}\right): \alpha^{n}\left(k_{0}, j_{0}\right)=(k \pm 1, *)\right\} .
\end{aligned}
$$

Then $A_{1}=A_{+} \cup A_{-}$and $A_{+} \cap A_{-}=\emptyset$. Now consider first the sum in (iv) over $A_{-}$. We get

$$
\begin{gathered}
\sum_{\left(k_{0}, j_{0}\right) \in \mathcal{A}_{-}}\left|\tilde{z}_{k_{0}, j_{0}}^{0}\right| \max _{1 \leq m \leq n+1}\left|\lambda_{\alpha^{m}\left(k_{0}, j_{0}\right)}^{0}-\lambda_{k_{0}, j_{0}}^{0}\right| \\
\leq \sum_{\left(k_{0}, j_{0}\right) \in \mathcal{A}_{-}}\left|\tilde{z}_{k_{0}, j_{0}}^{0}\right|\left(\left|\lambda_{\alpha^{n+1}\left(k_{0}, j_{0}\right)}^{n+1}-\lambda_{k_{0}, j_{0}}^{0}\right|\right. \\
\left.\cdot \bigvee_{1 \leq m \leq n} \max _{1 \leq m}\left|\lambda_{\alpha^{m}\left(k_{0}, j_{0}\right)}^{m}-\lambda_{k_{0}, j_{0}}^{0}\right|\right)
\end{gathered}
$$

where $a \vee b$ denotes the larger of $a$ and $b$. We have

$$
\begin{aligned}
& \sum_{\left(k_{0}, j_{0}\right) \in A_{-}}\left|\tilde{z}_{k_{0}, j_{0}}^{0}\right|\left(\left|\lambda_{\alpha^{n+1}\left(k_{0}, j_{0}\right)}^{n+1}-\lambda_{k_{0}, j_{0}}^{0}\right|\right) \\
& \quad \leq \sum_{\left(k_{0}, j_{0}\right) \in \mathcal{A}_{-}}\left|\tilde{z}_{k_{0}, j_{0}}^{0}\right|\left(\left|s\left(u_{l}, u_{r}\right)-\lambda_{k_{0}, j_{0}}^{0}\right|\right) \\
& \leq \sum_{\left(k_{0}, j_{0}\right) \in \mathcal{A}_{-}}\left|\tilde{z}_{k_{0}, j_{0}}^{0}\right|\left(\left|s\left(u_{l}, u_{r}\right)-s\left(u_{l}, u_{m}\right)\right|+\left|\lambda_{k-1, j}^{n}-\lambda_{k_{0}, j_{0}}^{0}\right|\right) \\
& \leq \sum_{\left(k_{0}, j_{0}\right) \in \mathcal{A}_{-}}\left|\tilde{z}_{k_{0}, j_{0}}^{0}\right|\left(K\left|u_{m}-u_{r}\right|+\left|\lambda_{k-1, j}^{n}-\lambda_{k_{0}, j_{0}}^{0}\right|\right) .
\end{aligned}
$$

Hence,

$$
\begin{aligned}
\sum_{\left(k_{0}, j_{0}\right) \in \mathcal{A}_{-}}\left|\tilde{z}_{k_{0}, j_{0}}^{0}\right| & \max _{1 \leq m \leq n+1}\left|\lambda_{\alpha^{m}\left(k_{0}, j_{0}\right)}^{0}-\lambda_{k_{0}, j_{0}}^{0}\right| \\
& \leq K \sum_{\left(k_{0}, j_{0}\right) \in \mathcal{A}_{-}}\left|\tilde{z}_{\alpha^{n}\left(k_{0}, j_{0}\right)}\right|\left|u_{m}-u_{r}\right|+\sigma \\
& \leq K\left|u_{m}-u_{r}\right|\left|u_{m}-u_{l}\right|+\sigma \leq K Q(\Delta)+\sigma .
\end{aligned}
$$

Since a similar estimate is valid for $A_{+}$, we see that (iv) holds.

Case (d). $R+S \rightarrow S$ (see Figure 8).

First note that $u_{m} \geq \bar{u}>u_{r}$. Now we obtain the partition of $\left[u_{r}, \bar{u}\right]$ by restriction of the partition of the interval $\left[u_{r}, u_{m}\right]$, retaining the classification. (Because of the construction of the original partitions, $\bar{u}$ is guaranteed to be a partition point of the interval $\left[u_{r}, u_{m}\right]$.) Set $\lambda_{k, j}^{n+1}=s\left(\bar{u}, u_{r}\right)$ for all $j$, and reclassify any wave $\tilde{z}_{k-1, j}^{n}$ between $\bar{u}$ and $u_{m}$ as $\tilde{\tilde{z}}_{k-1, j}^{n}$. (In fact, if $\alpha^{n}\left(k_{0}, j_{0}\right)=(k-1, j)$, then all the waves 


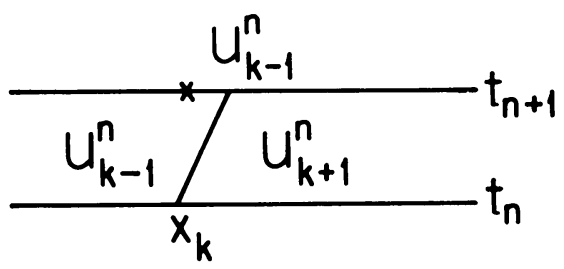

FIGURE 9

at $\alpha^{m}\left(k_{0}, j_{0}\right)$ for $0 \leq m \leq n$ get reclassified as $\tilde{\tilde{z}}$ 's.) We check (i): for any $m \leq n$ we reclassified waves of total strength at most $K\left|\bar{u}-u_{m}\right|$. For the diamond in Figure 8 we have

$C(\Delta)=\left|\bar{u}-u_{m}\right|+\left|u_{m}-u_{r}\right|-\left|\bar{u}-u_{r}\right|=\left(u_{m}-\bar{u}\right)+\left(u_{m}-u_{r}\right)-\left(\bar{u}-u_{r}\right)=2\left|u_{m}-\bar{u}\right|$.

(This is why the right side of (i) is $C\left(R^{p}\right)$ and not $C\left(R^{n}\right)$ !) Thus (i) holds. (iv) is verified as in the last case, and (ii) and (iii) are easy to check.

Case (e). $S+R \rightarrow S$. This is the same as in Case (d). Similarly, the cases $S+R \rightarrow R$ and $R+S \rightarrow R$ (other than translations) cannot occur, as we have noted above (after Figure 8). Since we have exhausted all cases, the proof is complete.

3. Estimate of the truncation error. Let $\phi$ be a nonnegative test function in $x-t$ space, and denote by $\beta(u)$ any Lipschitz-continuous function satisfying $\left|\beta^{\prime}(u)\right| \leq 1$ a.e. The truncation error is defined to be

$$
E(\phi) \equiv E(\phi, \beta, u)=\sum_{n=1}^{\infty} \int_{-\infty}^{\infty} \phi\left(x, t_{n}\right)\left[\beta\left(u\left(x, t_{n}\right)\right)\right] d x \equiv \sum_{n=1}^{\infty} E_{n}
$$

where $\left[f\left(\cdot, t_{n}\right)\right]$ denotes the jump in $f$ across the line $t=t_{n}$.

In order to estimate $E(\phi)$ we need some definitions and observations. Set $w_{k, j}^{n}=$ $\beta\left(y_{k, j}^{n}\right)-\beta\left(y_{k, j-1}^{n}\right)$; then the partition of the numbers $z_{k, j}^{n}=y_{k, j}^{n}-y_{k, j-1}^{n}$, defined in Theorem 2.4, can be extended to yield a partition of the numbers $w_{k, j}^{n}$ into disjoint sets $\left\{\tilde{w}_{k, j}^{n}\right\}$ and $\left\{\tilde{\tilde{w}}_{k, j}^{n}\right\}$. Note that

$$
\left|w_{k, j}^{n}\right| \leq\left|y_{k, j}^{n}-y_{k, j-1}^{n}\right|=\left|v_{k, j}^{n}\right| .
$$

We assume that the original partition on $t=0$ satisfies $\left|\lambda_{k, j}^{0}-\lambda_{k, j-1}^{0}\right| \leq \varepsilon$ for all $(k, j)$, where $\varepsilon>0$ is a given small number. We can now prove the following lemma.

LEMMA 3.1 .

$$
E_{n+1}=\sum_{k, j} \phi_{k}^{n+1}\left(\lambda_{k, j}^{n} \Delta t+\sigma_{k, j}^{n} \Delta x\right) w_{k, j}^{n}+O_{1}
$$

where

$$
O_{1} \leq K V \Delta t\left(\varepsilon\|\phi\|_{\infty}+\Delta x\left\|\phi_{x}\right\|_{\infty}\right),
$$

$K$ is a constant independent of $\Delta x$ and $\Delta t$, and $\sigma_{k, j}^{n}=\operatorname{sgn}\left(a^{n} \Delta x-\lambda_{k, j}^{n} \Delta t\right)$.

PROOF. Suppose that a shock wave emerges out of the point $\left(x_{k}, t_{n}\right)$ and $u_{k}^{n+1}=$ $u_{k-1}^{n}$; see Figure 9. 
In this case we have $\lambda_{k, j}^{n}=s=s\left(u_{k-1}^{n}, u_{k+1}^{n}\right)$ for all $j$, so that

$$
\begin{aligned}
& \sum_{j} \phi_{k}^{n+1}\left(\lambda_{k, j}^{n} \Delta t+\sigma_{k, j}^{n} \Delta x\right) w_{k, j}^{n}=\phi_{k}^{n+1}(s \Delta t-\Delta x) \sum_{j} w_{k, j}^{n} \\
& =\phi_{k}^{n+1}(s \Delta t-\Delta x)\left(\beta_{k+1}^{n}-\beta_{k-1}^{n}\right)=\phi_{k}^{n+1} \int_{x_{k-1}}^{x_{k+1}}[\beta] d x \\
& =\int_{x_{k-1}}^{x_{k+1}} \phi[\beta] d x,
\end{aligned}
$$

with an error

$$
\begin{aligned}
\int\left|\phi(x, t)-\phi\left(x_{k}, t\right)\right||[\beta]| d x & \leq\left\|\phi_{x}\right\|_{\infty} \Delta x \int|[\beta]| d x \\
& \leq\left.\left\|\phi_{x}\right\|_{\infty} V\right|_{\left[x_{k-1}, x_{k+1}\right]} \Delta x^{2}
\end{aligned}
$$

and this gives the desired result. The other case is that of a rarefaction wave emerging out of $\left(x_{k}, t_{n}\right)$; see Figure 10.

Suppose that $x_{k}+a_{n} \Delta x=x_{k}+\lambda_{k, j_{0}}^{n} \Delta t$, so that $u_{k}^{n+1}=y_{k, j_{0}}^{n}$. Then

$$
\sigma_{k, j}^{n}= \begin{cases}1 & \text { if } j \leq j_{0} \\ -1 & \text { if } j>j_{0}\end{cases}
$$

and using the notation $\beta_{k, j}^{n}=\beta\left(y_{k, j}^{n}\right)$, we have

$$
\begin{aligned}
& \sum_{j=1}^{q}\left(\lambda_{k, j}^{n} \Delta t+\sigma_{k, j}^{n} \Delta x\right) w_{k, j}^{n} \\
&=\sum_{j=1}^{q} \lambda_{k, j}^{n}\left(\beta_{k, j}^{n}-\beta_{k, j-1}^{n}\right) \Delta t+\sum_{j=1}^{j_{0}}\left(\beta_{k, j}^{n}-\beta_{k, j-1}^{n}\right) \Delta x \\
& \quad-\sum_{j=j_{0}+1}^{q}\left(\beta_{k, j}^{n}-\beta_{k, j-1}^{n}\right) \Delta x \\
&=\sum_{j=0}^{q-1} \beta_{k, j}^{n}\left(\lambda_{k, j}^{n}-\lambda_{k, j+1}^{n}\right) \Delta t+\left(\lambda_{k, q}^{n} \beta_{k, q}^{n}-\lambda_{k, 0}^{n} \beta_{k, 0}^{n}\right) \Delta t \\
&+\left(2 \beta_{k, j o}^{n}-\beta_{k, 0}^{n}-\beta_{k, q}^{n}\right) \Delta x \\
&= \sum_{j=0}^{q-1}\left(\beta_{k, j_{0}}^{n}-\beta_{k, j}^{n}\right)\left(\lambda_{k, j+1}^{n}-\lambda_{k, j}^{n}\right) \Delta t \\
&+\beta_{k, j_{0}}^{n}\left[\left(\lambda_{k, 0}^{n}-\lambda_{k, q}^{n}\right) \Delta t+2 \Delta x\right] \\
&+\beta_{k, q}^{n}\left(\lambda_{k, q}^{n} \Delta t-\Delta x\right)-\beta_{k, 0}^{n}\left(\lambda_{k, 0}^{n} \Delta t+\Delta x\right) \\
&= \sum_{j=0}^{q-1}\left(\beta_{k, j_{0}}^{n}-\beta_{k, j}^{n}\right)\left(\lambda_{k, j+1}^{n}-\lambda_{k, j}^{n}\right) \Delta t \\
&+\left(\beta_{k, j_{0}}^{n}-\beta_{k, q}^{n}\right)\left(\Delta x-\lambda_{k, q}^{n} \Delta t\right)+\left(\beta_{k, j_{0}}^{n}-\beta_{k, 0}^{n}\right)\left(\Delta x+\lambda_{k, q}^{n} \Delta t\right) \\
&= \int_{x_{k-1}}^{x_{k+1}}[\beta(x)] d x+E, \\
&
\end{aligned}
$$




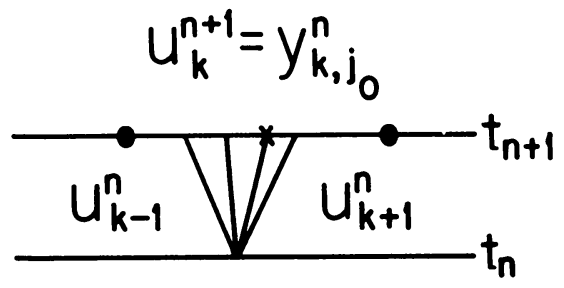

Figure 10

where

$$
\begin{aligned}
E & \leq \sum_{j=1}^{q}\left\{\left(\int_{x_{k}+\lambda_{k, j-1}^{n} \Delta t}^{x_{k}+\lambda_{k, j}^{n} \Delta t}[\beta(x)] d x\right)-[\beta]\left(x_{k}+\lambda_{k, j}^{n} \Delta t\right) \cdot\left(\lambda_{k, j}^{n}-\lambda_{k, j-1}^{n} \Delta t\right\}\right. \\
& \leq \sum_{j=1}^{q} \int_{x_{k}+\lambda_{k, j-1}^{n} \Delta t}^{x_{k}+\lambda_{k, j}^{n} \Delta t}\left|[\beta(x)]-[\beta]\left(x_{k}+\lambda_{k, j}^{n} \Delta t\right)\right| d x \\
& =\sum_{j=1}^{q} \int_{x_{k}+\lambda_{k, j-1}^{n} \Delta t}^{x_{k}+\lambda_{k, j}^{n} \Delta t}\left|\beta(x)-\beta\left(x_{k}+\lambda_{k, j}^{n} \Delta t\right)\right| d x \\
& \leq \sum_{j=1}^{q} \int_{x_{k}+\lambda_{k, j-1}^{n} \Delta t}^{x_{k}+\lambda_{k, j}^{n} \Delta t} \int_{x}^{x_{k}+\lambda_{k, j}^{n} \Delta t}\left|\beta^{\prime}(s)\right| d s d x \\
& \leq \sum_{j=1}^{q} \int_{x_{k}+\lambda_{k, j-1}^{n} \Delta t}^{x_{k}+\lambda_{k, j}^{n} \Delta t} \int_{x_{k}+\lambda_{k, j-1}^{n} \Delta t}^{x_{k}+\lambda_{k, j}^{n} \Delta t}\left|\beta^{\prime}(s)\right| d x d s \\
& =\sum_{j=1}^{q}\left(\lambda_{k, j}^{n}-\lambda_{k, j-1}^{n}\right) \Delta t \int_{x_{k}+\lambda_{k, j-1}^{n} \Delta t}^{x_{k}+\lambda_{k, j}^{n} \Delta t}\left|\beta^{\prime}(s)\right| d s \\
& \leq\left.\varepsilon \Delta t V\right|_{\left[x_{k-1}, x_{k+1}\right]},
\end{aligned}
$$

since we chose the partition to satisfy $\left|\lambda_{k, j}^{n}-\lambda_{k, j-1}^{n}\right| \leq \varepsilon$ for all pairs $(k, j)$ and each $\lambda_{k, j}^{n}-\lambda_{k, j-1}^{n}=\lambda_{p, q}^{0}-\lambda_{p, q-1}^{0}$ for some $(p, q)$. It follows that

$$
\begin{aligned}
\phi_{k}^{n+1} & \sum_{j=1}^{q}\left(\lambda_{k, j}^{n} \Delta t+\sigma_{k, j}^{n} \Delta x\right) w_{k, j}^{n} \\
& =\phi_{k}^{n+1} \int_{x_{k-1}}^{x_{k}}[\beta(x)] d x+O\left(\|\phi\|_{\infty} \varepsilon \Delta t V\right) .
\end{aligned}
$$

But

$$
\phi_{k}^{n+1} \int_{x_{k-1}}^{x_{k+1}}[\beta(x)] d x=\int_{x_{k-1}}^{x_{k+1}} \phi(x)[\beta(x)] d x,
$$

with an error which is bounded (as in the case of a shock), by

$$
\begin{aligned}
\int_{x_{k-1}}^{x_{k+1}}\left|\phi\left(x_{k}\right)-\phi(x)\right||[\beta(x)]| d x & \leq\left\|\phi_{x}\right\|_{\infty} \Delta x \int_{x_{k-1}}^{x_{k+1}}\left|\beta(x)-\beta\left(x_{k}+a_{n} \Delta x\right)\right| d x \\
& \leq\left\|\phi_{x}\right\|_{\infty} \Delta x \int_{x_{k-1}}^{x_{k+1}} \int_{x_{k-1}}^{x_{k+1}}\left|\beta^{\prime}(s)\right| d s d x \\
& \leq\left.\left\|\phi_{x}\right\|_{\infty} \Delta x^{2} V\right|_{\left[x_{k-1}, x_{k+1}\right]}
\end{aligned}
$$


This completes the proof of the lemma since $\Delta x=$ const $\Delta t$.

We now derive an estimate for the truncation error.

THEOREM 3.2. Let $E(\phi)$ be defined as in equation (3.1). Then for any Lipschitz-continuous function $\beta$ satisfying $\left|\beta^{\prime}(u)\right| \leq 1$ a.e.,

$$
|E(\phi)| \leq K\left[\Delta t^{1 / 2}|\log \Delta t|\|\phi\|_{\infty}+\Delta t\|\nabla \phi\|_{\infty}\right],
$$

where $K$ is a constant independent of $\Delta t, \Delta x$, and $\beta$.

PrOOF. Recall that in $\S 2$ we partitioned $\mathbf{Z}_{+}$(the time-line indices) into sets of equal length $\{(p-1) M \leq n<p M\}, p=1,2, \ldots$, which define strips in $t>0$. We shall fix an $n$ in a given strip and refine our estimate of $E_{n}$. For simplicity in notation we pick an $n$ in the first strip $(p=1)$, make our estimates on the corresponding $E_{n}$, and then sum over all $n$ in the first strip. Since the result we get is the same for all strips, this will enable us to obtain the desired estimate on $E(\phi)$. Next, if we delete all of the $\tilde{\tilde{w}}$ terms occurring in the $E_{n+1}$ equation in the statement of Lemma 3.1, then $|w| \leq|v|$ (because $\beta$ is Lipschitz continuous with Lipschitz constant 1), and so from Theorem 2.4(i), we commit an error of

$$
K\|\phi\|_{\infty} \Delta t C\left(R^{1}\right) \equiv O_{2} .
$$

We now consider only the $\tilde{w}$ terms and, for simplicity in notation, will omit the tildes. We have

$$
\begin{aligned}
E_{n+1} & =\sum_{k, j} \phi_{k}^{n+1}\left(\lambda_{k, j}^{n} \Delta t+\sigma_{k, j}^{n} \Delta x\right) w_{k, j}^{n}+O_{1}+O_{2} \\
& =\sum_{(k, j) \in N} \phi_{\alpha^{n}(k, j)}^{n+1}\left(\lambda_{\alpha^{n}(k, j)}^{n} \Delta t+\sigma_{\alpha^{n}(k, j)}^{n}\right) w_{\alpha_{n}(k, j)}^{n}+O_{1}+O_{2},
\end{aligned}
$$

where $N=\left\{(k, j): \tilde{\tilde{z}}_{k, j}^{0}=0\right\}$. Thus,

$$
\begin{aligned}
E_{n+1}=\sum_{N} \phi_{\alpha^{n}(k, j)}^{n+1}[ & \left(\lambda_{k, j}^{0} \Delta t+\sigma_{\alpha^{n}(k, j)}^{n} \Delta x\right) w_{k, j}^{0} \\
& +\left(\lambda_{\alpha^{n}(k, j)}^{n}-\lambda_{k, j}^{0}\right) w_{\alpha^{n}(k, j)}^{n} \Delta t \\
& \left.+\left(\lambda_{k, j}^{0} \Delta t+\sigma_{\alpha^{n}(k, j)}^{n} \Delta x\right)\left(w_{\alpha^{n}(k, j)}^{n}-w_{k, j}^{0}\right)\right]+O_{1}+O_{2} .
\end{aligned}
$$

Now from Lemma 2.4(iii),

$$
w_{\alpha^{n}(k, j)}^{n}-w_{k, j}^{0}=\beta\left(y_{\alpha^{n}(k, j)}^{n}\right)-\beta\left(y_{k, j}^{0}\right)=0 .
$$

Also, using Lemma 2.4(iv),

$$
\begin{aligned}
\sum_{N}\left|\lambda_{\alpha^{n}(k, j)}^{n}-\lambda_{k, j}^{0}\right|\left|w_{\alpha^{n}(k, j)}^{n}\right| \phi_{\alpha^{n}(k, j)}^{n+1} \Delta t \\
\leq\|\phi\|_{\infty} \sum_{k, j}\left|v_{\alpha^{n}(k, j)}^{n}\right|\left|\lambda_{\alpha^{n}(k, j)}^{n}-\lambda_{k, j}^{0}\right| \Delta t \\
=\|\phi\|_{\infty} \sum_{k, j}\left|v_{k, j}^{0}\right|\left|\lambda_{\alpha^{n}(k, j)}^{n}-\lambda_{k, j}^{0}\right| \Delta t \\
\leq K\|\phi\|_{\infty} Q\left(R^{1}\right) \Delta t .
\end{aligned}
$$


Thus setting

$$
O_{3}=K\|\phi\|_{\infty} Q\left(R^{1}\right) \Delta t
$$

we have, from (3.4)-(3.6),

$$
\begin{aligned}
E_{n+1} & =\sum_{N} \phi_{\alpha^{n}(k, j)}^{n+1}\left(\lambda_{k, j}^{0} \Delta t+\sigma_{\alpha^{n}(k, j)}^{n} \Delta x\right) w_{k, j}^{0}+O_{1}+O_{2}+O_{3} \\
& =\sum_{N} \phi_{k}^{0}\left(\lambda_{k, j}^{0} \Delta t+\sigma_{\alpha^{n}(k, j)}^{n} \Delta x\right) w_{k, j}^{0}+O_{1}+O_{2}+O_{3}+O_{4}
\end{aligned}
$$

where

$$
\|\nabla \phi\|_{\infty} M \Delta t \sum_{N}\left|w_{k, j}^{n}\right| \Delta t \leq K\|\nabla \phi\|_{\infty} M \Delta t^{2} V \equiv O_{4} .
$$

We next estimate the total error terms; namely,

$$
\begin{aligned}
& \sum_{n=1}^{M}\left(O_{1}+O_{2}+O_{3}+O_{4}\right) \\
& \leq K V M \Delta t\left(\varepsilon\|\phi\|_{\infty}+\Delta x\|\nabla \phi\|_{\infty}\right)+K M\|\phi\|_{\infty} \Delta t C\left(R^{1}\right) \\
&+K M\|\phi\|_{\infty} \Delta t Q\left(R^{1}\right)+K\|\nabla \phi\|_{\infty} M \Delta t^{2} V \\
&= K\|\phi\|_{\infty} M \Delta t\left(\varepsilon V+C\left(R^{1}\right)+Q\left(R^{1}\right)\right)+K\|\nabla \phi\|_{\infty} M \Delta t^{2} V \equiv O_{5}
\end{aligned}
$$

Therefore, summing over the first strip,

$$
\begin{aligned}
\sum_{n=1}^{M} E_{n}= & \sum_{N} \sum_{n=1}^{M} \phi_{k}^{0}\left(\lambda_{k, j}^{0} \Delta t+\sigma_{\alpha^{n}(k, j)}^{n} \Delta x\right) w_{k, j}^{0}+O_{5} \\
= & \sum_{k} \phi_{k}^{0} \sum_{j} \sum_{n=1}^{M}\left(\lambda_{k, j}^{0} \Delta t+\bar{\sigma}_{k, j}^{n} \Delta x\right) w_{k, j}^{0} \\
& +\sum_{k} \phi_{k}^{0} \sum_{j} \sum_{n=1}^{M}\left(\sigma_{\alpha^{n}(k, j)}^{n}-\bar{\sigma}_{k, j}^{n}\right) \Delta x w_{k, j}^{0}+O_{5}
\end{aligned}
$$

where

$$
\bar{\sigma}_{k, j}^{n}=\operatorname{sgn}\left(a_{n} \Delta x-\lambda_{k, j}^{0} \Delta t\right) .
$$

Note that

$$
\sigma_{\alpha^{n}(k, j)}^{n}=\operatorname{sgn}\left(a_{n} \Delta x-\lambda_{\alpha^{n}(k, j)}^{n} \Delta t\right),
$$

so that $\left|\bar{\sigma}_{k, j}^{n}-\sigma_{\alpha^{n}(k, j)}^{n}\right|=0$, unless $a_{n} \Delta x$ lies between $\lambda_{k, j}^{0} \Delta t$ and $\lambda_{\alpha^{n}(k, j)}^{n}$. It follows that $\sum_{n=1}^{M}\left|\bar{\sigma}_{k, j}^{n}-\sigma_{\alpha^{n}(k, j)}^{n}\right|$ is at most the number of $n, 1 \leq n \leq M$, such that

$$
\min _{0 \leq m \leq M} \lambda_{\alpha^{m}(k, j)}^{m} \Delta t \leq a_{n} \Delta x \leq \max _{0 \leq m \leq M} \lambda_{\alpha^{m}(k, j)}^{m} \Delta t .
$$

Let $I_{k, j}$ be the interval

$$
I_{k, j}=\left[\min _{0 \leq m \leq M} \lambda_{\alpha^{m}(k, j)}^{m}, \max _{0 \leq m \leq M} \lambda_{\alpha^{m}(k, j)}^{m}\right] .
$$


From (1.4), we have

$$
\begin{aligned}
\sum_{n=1}^{M}\left|\bar{\sigma}_{k, j}^{n}-\sigma_{\alpha^{n}(k, j)}^{n}\right| & \leq \#\left\{n: 1 \leq n \leq M \text { and } a_{n} \in I_{k, j}\right\} \\
& \leq K\left(M\left|I_{k, j}\right|+\log M\right) .
\end{aligned}
$$

Thus (cf. (3.10)),

$$
\begin{aligned}
& \left|\sum_{k} \phi_{k}^{0} \sum_{j} \sum_{n=1}^{M}\left(\sigma_{\alpha^{n}(k, j)}-\bar{\sigma}_{k, j}\right) \Delta x w_{k, j}^{0}\right| \\
& \leq K\|\phi\|_{\infty} \sum_{(k, j) \in N}\left(M\left|I_{k, j}\right|+\log M\right)\left|w_{k, j}^{0}\right| \Delta x \\
& \leq K\|\phi\|_{\infty} \Delta x\left(M Q\left(R^{1}\right)+V \log M\right),
\end{aligned}
$$

where we have used Theorem 2.4(iv) in order to obtain the last inequality. If we define

$$
\begin{aligned}
O_{6} & =O_{5}+\|\phi\|_{\infty} \Delta x\left(M Q\left(R^{1}\right)+V \log M\right) \\
& =K\|\phi\|_{\infty} M \Delta t\left[\varepsilon V+C\left(R^{1}\right)+(\log M) / M\right]+K\|\nabla \phi\|_{\infty} M \Delta t^{2} V
\end{aligned}
$$

then we have from (3.10),

$$
\sum_{n=1}^{M} E_{n}=\sum_{(k, j) \in N} \phi_{k}^{0} w_{k, j}^{0} \sum_{n=1}^{M}\left(\lambda_{k, j}^{0} \Delta t+\bar{\sigma}_{k, j}^{n} \Delta x\right)+O_{6}
$$

We now estimate $\sum_{n=1}^{M}\left(\lambda_{k, j}^{0} \Delta t+\bar{\sigma}_{k, j}^{n} \Delta x\right)$. To this end, note that using (1.4) again, we have

$$
\begin{aligned}
\sum_{n=1}^{M} \frac{1-\bar{\sigma}_{k, j}^{n}}{2} & =\sum_{n=1}^{M} \frac{1-\operatorname{sgn}\left(a_{n} \Delta x-\lambda_{k, j}^{0} \Delta t\right)}{2} \\
& =\#\left\{n: 1 \leq n \leq M \text { and }-1 \leq a_{n} \leq \lambda_{k, j}^{0} \Delta t / \Delta x\right\} \\
& =M\left[\frac{1+\lambda_{k, j}^{0} \Delta t / \Delta x}{2}+O\left(\frac{\log M}{M}\right)\right] \equiv B
\end{aligned}
$$

Thus if $I=\left[-1, \lambda_{k, j}^{0} \Delta t / \Delta x\right]$, we have

$$
\sum_{n=1}^{M} \bar{\sigma}_{k, j}^{n}=M-2 B
$$

and so

$$
\begin{aligned}
\sum_{n=1}^{M}\left(\lambda_{k, j}^{0} \Delta t+\bar{\sigma}_{k, j}^{n} \Delta x\right) & =M \lambda_{k, j}^{0} \Delta t+M \Delta x-2 \Delta x B \\
& =2 M \Delta x\left[\frac{\lambda_{k, j}^{0} \Delta t / \Delta x+1}{2}-\frac{B}{M}\right] \\
& =2 M \Delta x\left[\frac{|I|}{2}-\frac{B}{M}\right] \leq 2 M \Delta x K \frac{\log M}{M}=K \Delta x \log M,
\end{aligned}
$$


where we have used (3.11). Thus from (3.13),

$$
\begin{aligned}
\sum_{n=1}^{M} E_{n} & \leq K\|\phi\|_{\infty} \sum_{(k, j) \in N}\left|w_{k, j}^{0}\right| \Delta x \log M+O_{6} \\
& \leq K V\|\phi\|_{\infty} \Delta t \log M+O_{6} .
\end{aligned}
$$

Now using this together with (3.12), we have, as $\varepsilon \rightarrow 0$,

$$
\begin{aligned}
\sum_{n=1}^{M} E_{n} \leq & K V\|\phi\|_{\infty} M \Delta t\left[C\left(R^{1}\right)+Q\left(R^{1}\right)+\frac{\log M}{M}\right] \\
& +K M\|\nabla \phi\|_{\infty} V \Delta t^{2},
\end{aligned}
$$

where $K$ is a constant independent of the mesh parameters $\Delta t, \Delta x$.

Now considering the general case where $p>1$, it is easy to see that (3.14) goes over into

$$
\begin{aligned}
\sum_{n=(p-1) M}^{p M} E_{n} \leq & K V\|\phi\|_{\infty} M \Delta t\left[C\left(R^{p}\right)+Q\left(R^{p}\right)+\frac{\log M}{M}\right] \\
& +K M\|\nabla \phi\|_{\infty} V \Delta t^{2} .
\end{aligned}
$$

If $\operatorname{spt} \phi \subseteq\{0 \leq t \leq T\}$ and $N M \Delta t=T$, where $N \in \mathbf{Z}_{+}$, then summing (3.15) over all $p$ between 1 and $N$ gives

$$
\sum_{n=1}^{\infty} E_{n} \leq K\|\phi\|_{\infty} M \Delta t\left(1+\frac{N \log M}{M}\right)+K M N\|\nabla \phi\|_{\infty} \Delta t^{2} .
$$

Thus, if we choose $M=O\left(\Delta t^{-1 / 2}\right)$, we finally obtain

$$
|E(\phi)| \leq K\|\phi\|_{\infty}\left(\Delta t^{1 / 2}+\Delta t^{1 / 2}|\log \Delta t|\right)+K\|\nabla \phi\|_{\infty} \Delta t,
$$

as desired. This completes the proof.

4. The error bound. In order to derive the error bound (1.3), we shall prove a slight refinement of the following theorem due to Krushkov [K].

THEOREM. Let $w_{0} \in L^{\infty}(\mathbf{R})$ be given. Then there is a unique solution $w$ of (1.1)-(1.2) satisfying

$$
\iint_{t \geq 0}\left\{|w-k| \phi_{t}+\operatorname{sgn}(w-k)[f(w)-f(k)]\right\} d x d t \geq 0
$$

for any nonnegative test function supported in $\{t>0\}$ and any constant $k$. $w$ achieves the initial value $w_{0}$ in the sense that $w(t) \rightarrow w_{0}$ in $L_{\text {loc }}^{1}(\mathbf{R})$ as $t \rightarrow 0$.

For our purposes we consider the above double integral when boundary terms can occur; i.e., when the support of $\phi$ meets $t=0$. Here is the result.

LEMMA 4.1. Let $\phi=\phi(x, t)$ be a nonnegative, Lipschitz-continuous function supported in a strip $x_{1} \leq x \leq x_{2}$, and let $w$ be a function which is an exact solution 


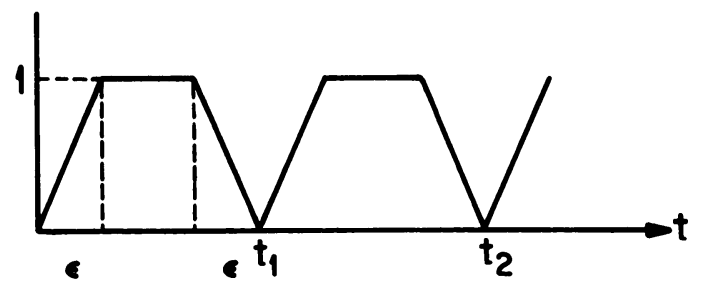

Figure 11

in the open strips $t_{n}<t<t_{n+1}, n=0,1, \ldots, N-1$. If $T=t_{N}$, then for any constant $k$,

$$
\begin{aligned}
\int_{0}^{T} & \int_{-\infty}^{\infty}\left\{|w-k| \phi_{t}+\operatorname{sgn}(w-k)[f(w)-f(k)] \phi_{x}\right\} d x d t \\
& \geq\left.\int_{-\infty}^{\infty}|w-k| \phi\right|_{t=T-} d x-\left.\int_{-\infty}^{\infty}|w-k| \phi\right|_{t=0} d x \\
& -\left.\sum_{n=1}^{N-1} \int_{-\infty}^{\infty}[|w-k| \phi]\right|_{t=t_{n}-} ^{t=t_{n}+} d x
\end{aligned}
$$

Proof. Let $\varepsilon>0$ be small, and let $\chi_{\varepsilon}(t)$ be the piecewise linear (periodic) function depicted in Figure 11.

If

then

$$
\psi_{\varepsilon}(x, t)= \begin{cases}\chi_{\varepsilon}(t) \phi(x, t), & t_{n} \leq t \leq t_{n+1}, \\ 0, & \text { otherwise }\end{cases}
$$

$$
\begin{aligned}
0 \leq & \int_{t_{n}}^{t_{n+1}} \int\left\{|w-k|\left(\psi_{\varepsilon}\right)_{t}+\operatorname{sgn}(w-k)[f(w)-f(k)] \chi_{\varepsilon} \phi_{x}\right\} d x d t \\
= & \int_{t_{n}}^{t_{n+1}} \int|w-k| \chi_{\varepsilon} \phi_{t} d x d t \\
& +\frac{1}{\varepsilon}\left(\int_{t_{n}}^{t_{n}+\varepsilon} \int-\int_{t_{n+1}-\varepsilon}^{t_{n+1}} \int\right)|w-k| \phi_{t} d x d t \\
& +\int_{t_{n}}^{t_{n+1}} \int \operatorname{sgn}(w-k)[f(w)-f(k)] \chi_{\varepsilon} \phi_{x} d x d t .
\end{aligned}
$$

Thus summing over $n$ gives

$$
\begin{aligned}
0 \leq & \int_{0}^{T} \int\left\{|w-k| \phi_{t}+\operatorname{sgn}(w-k)[f(w)-f(k)] \phi_{x}\right\} \chi_{\varepsilon} d x d t \\
& +\frac{1}{\varepsilon} \sum_{n=1}^{N}\left(\int_{t_{n-1}}^{t_{n-1}+\varepsilon} \int-\int_{t_{n}-\varepsilon}^{t_{n}} \int\right)|w-k| \phi_{t} d x d t .
\end{aligned}
$$

But

$$
\begin{array}{r}
\lim _{\varepsilon \rightarrow 0} \int_{0}^{T} \int\left\{|w-k| \phi_{t}+\operatorname{sgn}(w-k)[f(w)-f(k)] \phi_{x}\right\} \chi_{\varepsilon} d x d t \\
\quad=\int_{0}^{T} \int\left\{|w-k| \phi_{t}+\operatorname{sgn}(w-k)[f(w)-f(k)] \phi_{x}\right\} d x d t
\end{array}
$$


and since the evaluation map

$$
\left.t \rightarrow \int_{-\infty}^{\infty}|w-k| \phi_{t}\right|_{t} d x
$$

is Lipschitz continuous on $\left[t_{n}, t_{n-1}\right]$, we see that if we let $\varepsilon \rightarrow 0$ in (4.1), we get

$$
\begin{aligned}
0 \leq \int_{0}^{T} \int\left\{|w-k| \phi_{t}+\operatorname{sgn}(w-k)[f(w)-f(k)] \phi_{x}\right\} d x d t \\
\quad+\left.\sum_{n=0}^{N-1} \int|w-k| \phi_{t}\right|_{t_{n+1^{-}}} ^{t_{n+1^{+}}} d x \\
=\int_{0}^{T} \int\left\{|w-k| \phi_{t}+\operatorname{sgn}(w-k)[f(w)-f(k)] \phi_{x}\right\} d x d t \\
\quad-\left.\int|w-k| \phi_{t}\right|_{T-}+\left.\int|w-k| \phi_{t}\right|_{0+}+\left.\sum_{n=1}^{N-1} \int\left[|w-k| \phi_{t}\right]\right|_{t_{n^{-}}} ^{t_{n^{+}}},
\end{aligned}
$$

and this gives the desired result.

We are now in a position to derive the error bounds. Let $\phi(x, t, y, \tau)$ be a nonnegative test function having compact support in $(x, y)$-space. Let $T=t_{N}$ as before. If $u$ denotes a Glimm approximate solution, and $v$ is the exact solution of (1.1), (1.2), then taking $\beta=|u-v(y, \tau)|$ in Theorem 3.2 and using the last lemma, we can write

$$
\begin{aligned}
\int_{0}^{\infty} \int_{-\infty}^{\infty}\left\{|u(x, t)-v(y, \tau)| \phi_{t}+\operatorname{sgn}(u(x, t)-v(y, \tau))\right. \\
\left.\cdot[f(u(x, t))-f(v(y, \tau))] \phi_{x}\right\} d x d t \\
\geq \int_{-\infty}^{\infty}|u(x, T)-v(y, \tau)| \phi(x, T, y, \tau) d x \\
\quad-\int_{-\infty}^{\infty}|u(x, 0)-v(y, \tau)| \phi(x, 0, y, \tau) d x-E(\phi, \beta, u) \\
\geq \int_{-\infty}^{\infty}|u(x, T)-v(y, \tau)| \phi(x, T, y, \tau) d x \\
=\int_{-\infty}^{\infty}|u(x, 0)-v(y, \tau)| \phi(x, 0, y, \tau) d x \\
\quad-K\left(\Delta t^{1 / 2}|\log \Delta t|\|\phi\|_{\infty}+\Delta t\left\|\nabla_{x, t} \phi\right\|_{\infty}\right) .
\end{aligned}
$$

If we integrate this inequality over the support of $\phi$ in $y$ space cross the interval $[0, T]$, we get (omitting the limits of integration),

$$
\begin{aligned}
\iiint \int & \left\{|u-v| \phi_{t}+\operatorname{sgn}(u-v)[f(u)-f(v)] \phi_{x}\right\} d x d t d y d \tau \\
\geq & \iiint|u(x, T)-v(y, \tau)| \phi(x, T, y, \tau) d x d y d \tau \\
& \quad-\iiint|u(x, 0)-v(y, \tau)| \phi(x, 0, y, \tau) d x d y d \tau-E_{1}
\end{aligned}
$$

where

$$
E_{1}=O\left(\Delta t^{1 / 2}|\log \Delta t|\|\phi\|_{\infty}+\Delta t\|\nabla \phi\|_{\infty}\right) .
$$


Using Lemma 4.1 we find similarly,

$$
\begin{gathered}
\iiint \int\left\{|u-v| \phi_{\tau}+\operatorname{sgn}(u-v)[f(u)-f(v)] \phi_{y}\right\} d x d t d y d \tau \\
\geq \iiint|v(y, T)-u(x, t)| \phi(x, t, y, T) d y d x d t \\
\quad-\iiint|v(y, 0)-u(x, t)| \phi(x, t, y, 0) \mid d y d x d t
\end{gathered}
$$

Thus, adding (4.2) and (4.3) gives

$$
\begin{aligned}
\iiint \int & \left\{|u(x, t)-v(y, \tau)|\left(\phi_{t}+\phi_{\tau}\right)\right. \\
& \left.\quad+\operatorname{sgn}(u-v)[f(u)-f(v)]\left(\phi_{x}+\phi_{y}\right)\right\} d x d t d y d \tau \\
\geq & \iiint|u(x, T)-v(y, \tau)| \phi(x, T, y, \tau) d x d y d \tau \\
\quad & -\iiint|u(x, 0)-v(y, \tau)| \phi(x, 0, y, \tau) d x d y d \tau \\
\quad+\iiint|u(x, t)-v(y, T)| \phi(x, t, y, T) d x d y d t & \\
\quad & -\iiint|u(x, t)-v(y, 0)| \phi(x, t, y, 0) d x d y d t-K E_{1}
\end{aligned}
$$

We want to estimate the difference between these integrals and integrals on the diagonal. To this end, we follow $[\mathbf{K}]$ and define

$$
\phi(x, t, y, \tau)=\psi\left(\frac{x+y}{2}, \frac{t+\tau}{2}\right) \delta_{h}\left(\frac{x-y}{2}, \frac{t-\tau}{2}\right)
$$

where $\psi$ is a nonnegative test function with $\psi(\cdot, s)$ compact for each $s>0$, and $\delta_{h}$ is an approximate "delta function". That is,

$$
\delta_{h}(y, t)=\delta(y / h) \delta(t / h) h^{-2}
$$

where $\delta \geq 0, \int_{-\infty}^{\infty} \delta(s) d s=1$, and the support of $\delta$ lies in the interval $(-1,1)$. For this choice of $\phi$,

$$
\begin{aligned}
E_{1} & \sim\|\psi\|_{\infty} h^{-2} \Delta t^{1 / 2}|\log \Delta t|+\|\nabla \psi\|_{\infty} h^{-2} \Delta t+\|\psi\|_{\infty} h^{-3} \Delta t \\
& =\|\psi\|_{\infty}\left(h^{-2} \Delta t^{1 / 2}|\log \Delta t|+h^{-3} \Delta t\right)+\|\nabla \psi\|_{\infty} h^{-2} \Delta t \equiv E_{2}
\end{aligned}
$$


and (4.4) becomes

$$
\begin{aligned}
\iiint \int & \left\{u(x, t)-v(y, \tau) \mid \psi_{t}\left(\frac{x+y}{2}, \frac{t+\tau}{2}\right)\right. \\
& \left.+\operatorname{sgn}(u-v)[f(u)-f(v)] \psi_{x}\right\} \delta_{h}\left(\frac{x-y}{2}, \frac{t-\tau}{2}\right) \\
\geq & \iiint|u(x, T)-v(y, \tau)| \psi\left(\frac{x+y}{2}, \frac{T+\tau}{2}\right) \delta_{h}\left(\frac{x-y}{2}, \frac{T-\tau}{2}\right) d x d y d \tau \\
& -\iiint|u(x, 0)-v(y, \tau)| \psi\left(\frac{x+y}{2}, \frac{\tau}{2}\right) \delta_{h}\left(\frac{x-y}{2}, \frac{-\tau}{2}\right) d x d y d \tau \\
& +\iiint|u(x, t)-v(y, T)| \psi\left(\frac{x+y}{2}, \frac{T+\tau}{2}\right) \delta_{h}\left(\frac{x-y}{2}, \frac{T-t}{2}\right) d x d y d t \\
& -\iiint|u(x, t)-v(y, 0)| \psi\left(\frac{x+y}{2}, \frac{t}{2}\right) \delta_{h}\left(\frac{x-y}{2}, \frac{t}{2}\right) d x d y d t-E_{2} \\
\equiv & \tilde{I}_{3}-I_{3}+\tilde{I}_{4}-I_{4}-E_{2} .
\end{aligned}
$$

We now estimate the difference between these integrals and integrals on the diagonal. To this end, let

$$
I_{1}=\iiint \int|u(x, t)-v(y, \tau)| \psi_{t}\left(\frac{x+y}{2}, \frac{t+\tau}{2}\right) \delta_{h}\left(\frac{x-y}{2}, \frac{t-\tau}{2}\right) .
$$

Then

$$
\begin{aligned}
I_{1} & =\iiint \int|u(x, t)-v(x, t)| \psi_{t}(x, t) \delta_{h}\left(\frac{x-y}{2}, \frac{t-\tau}{2}\right) d x d t d y d \tau+E_{3} \\
& =\iint|u-v| \psi_{t} d x d t+E_{3}
\end{aligned}
$$

where

(4.7)

$$
\begin{aligned}
& E_{3}= \iiint \int[|u(x, t)-v(y, \tau)|-|u(x, t)-v(x, t)|] \\
& \cdot \psi_{t}\left(\frac{x+y}{2}, \frac{t+\tau}{2}\right) \delta_{h}\left(\frac{x-y}{2}, \frac{t-\tau}{2}\right) \\
& \quad+\iiint \int|u(x, t)-v(x, t)|\left[\psi_{t}\left(\frac{x+y}{2}, \frac{t+\tau}{2}\right)-\psi_{t}(x, t)\right] \delta_{h}\left(\frac{x-y}{2}, \frac{t-\tau}{2}\right) \\
& \equiv J_{1}+J_{2} .
\end{aligned}
$$

Now

$$
\begin{aligned}
J_{1} & \leq \iiint \int|v(y, \tau)-v(x, t)|\left|\psi_{t}\left(\frac{x+y}{2}, \frac{t+\tau}{2}\right)\right| \delta_{h}\left(\frac{x-y}{2}, \frac{t-\tau}{2}\right) \\
& \leq\left\|\psi_{t}\right\|_{\infty} \iiint \int[|v(y, \tau)-v(y, t)|+|v(y, t)-v(x, t)|] \delta_{h}\left(\frac{x-y}{2}, \frac{t-\tau}{2}\right),
\end{aligned}
$$


and

$$
\begin{array}{r}
\iiint \int|v(y, t)-v(x, t)| \delta_{h}\left(\frac{x-y}{2}, \frac{t-\tau}{2}\right) d \tau d y d t d x \\
=\iiint \int|v(y, t)-v(x, t)| \delta_{h}\left(\frac{x-y}{2}\right) \delta_{h}\left(\frac{t-\tau}{2}\right) \\
\quad=\int_{0}^{T} \int_{-\infty}^{\infty} \int_{x-h}^{x+h}|v(y, t)-v(x, t)| \delta_{h}\left(\frac{x-y}{2}\right) d y d x d t
\end{array}
$$

since

$$
\int_{0}^{T} \delta_{h}\left(\frac{t-\tau}{2}\right) d \tau=1
$$

Now interpreting $v_{x}$ as a measure having finite total variation, we have

$$
\begin{aligned}
\int_{0}^{T} & \int_{-\infty}^{\infty} \int_{x-h}^{x+h}|v(y, t)-v(x, t)| \delta_{h}\left(\frac{x-y}{2}\right) d y d x d t \\
& \leq \int_{0}^{T} \int_{-\infty}^{\infty} \int_{x-h}^{x+h} \int_{x-h}^{x+h}\left|v_{x}(s, t)\right| \delta_{h}\left(\frac{x-y}{2}\right) d s d y d x d t \\
& =\int_{0}^{T} \int_{-\infty}^{\infty}\left[\left(\int_{x-h}^{x+h} \delta_{h}\left(\frac{x-y}{2}\right) d y\right)\left(\int_{x-h}^{x+h}\left|v_{x}(s, t)\right| d s\right)\right] d x d t \\
& \leq \int_{0}^{T} \int_{-\infty}^{\infty} \int_{x-h}^{x+h}\left|v_{x}(s, t)\right| d s d x d t \\
& =\int_{0}^{T} \int_{-\infty}^{\infty} \int_{s-h}^{s+h}\left|v_{x}(s, t)\right| d x d s d t \\
& =2 h \int_{0}^{T} \int_{-\infty}^{\infty}\left|v_{x}(s, t)\right| d s d t \\
& =2 h \int_{0}^{T}(\operatorname{Tot} . \operatorname{Var} . v(\cdot, t)) d t \\
& =2 h T V
\end{aligned}
$$

where, we recall, $V=$ Tot. Var. $v(\cdot, 0)$. The last inequality follows from Lemma 2.1. Hence,

$$
\iiint \int|v(y, t)-v(x, t)| \delta_{h}\left(\frac{x-y}{2}, \frac{t-\tau}{2}\right) d \tau d y d x d t \leq 2 h T V .
$$

Similarly, using the fact that $v_{t}=-f(u)_{x}$ (in the sense of measures), we find

$$
\iiint \int|v(y, \tau)-v(y, t)| \delta_{h}\left(\frac{x-y}{2}, \frac{t-\tau}{2}\right) d \tau d y d x d t \leq \text { const } h .
$$

It follows that

$$
\left|J_{1}\right| \leq K\left\|\psi_{t}\right\|_{\infty} h
$$


We next estimate $J_{2}$. We have

$$
\begin{aligned}
\left|J_{2}\right| & \leq\left\|\nabla \psi_{t}\right\|_{\infty} h \iint|u(x, t)-v(x, t)|\left(\iint \delta_{h}\left(\frac{x-y}{2}, \frac{t-\tau}{2}\right) d y d \tau\right) d x d t \\
& =\left\|\nabla \psi_{t}\right\|_{\infty} h \iint|u(x, t)-v(x, t)| d x d t \\
& \leq\left\|\nabla \psi_{t}\right\|_{\infty} h\|u-v\|_{L^{1}(\mathrm{spt} \psi)} .
\end{aligned}
$$

Now referring to (4.6), we observe that we can treat the term

$$
\iiint \int \operatorname{sgn}(u-v)[f(u)-f(v)] \psi_{x} \delta_{h}\left(\frac{x-y}{2}, \frac{t-\tau}{2}\right)
$$

in a similar way, so we get

(4.10)

$$
\begin{aligned}
\iint\left\{|u(x, t)-v(x, t)| \psi_{t}+\operatorname{sgn}(u-v)[f(u)-f(v)] \psi_{x}\right\} d x d t \\
\quad \geq \tilde{I}_{3}-I_{3}+\tilde{I}_{4}-I_{4}-E_{2}-K\left(\left\|\psi_{t}\right\|_{\infty} h+\left\|\nabla \psi_{t}\right\|_{\infty} h\|u-v\|_{1}\right) \\
\quad=\tilde{I}_{3}-I_{3}+\tilde{I}_{4}-I_{4} \\
\quad-K\left[\|\psi\|_{\infty} h^{-2} \Delta t^{1 / 2}|\log \Delta t|+\|\nabla \psi\|_{\infty}\left(h^{-2} \Delta t+h\right)+\left\|\nabla \psi_{t}\right\|_{\infty}\|u-v\|_{1} h\right] \\
\quad \equiv \tilde{I}_{3}-I_{3}+\tilde{I}_{4}-I_{4}-E_{4} .
\end{aligned}
$$

Now let us consider $I_{3}$ :

$$
\begin{aligned}
I_{3} & =\iiint|u(x, 0)-v(y, \tau)| \psi\left(\frac{x+y}{2}, \frac{\tau}{2}\right) \delta_{h}\left(\frac{x-y}{2}, \frac{-\tau}{2}\right) d x d y d \tau \\
& =\iiint|u(x, 0)-v(x, 0)| \psi(x, 0) \delta_{h}\left(\frac{x-y}{2}, \frac{-\tau}{2}\right) d x d y d \tau+e \\
& =\int \mid u(x, 0)-v(x, 0) \psi(x, 0) d x+e
\end{aligned}
$$

where

$$
\begin{aligned}
e= & \iiint[|u(x, 0)-v(y, \tau)|-|u(x, 0)-v(x, 0)|] \psi\left(\frac{x+y}{2}, \frac{-\tau}{2}\right) \delta_{h}\left(\frac{x-y}{2}, \frac{\tau}{2}\right) \\
& +\iiint|u(x, 0)-v(x, 0)|\left[\psi\left(\frac{x+y}{2}, \frac{-\tau}{2}\right)-\psi(x, 0)\right] \delta_{h}\left(\frac{x-y}{2}, \frac{\tau}{2}\right) .
\end{aligned}
$$

If we compare the error term $e$ with (4.7) we see that we can estimate this term in a manner analogous to the way we just estimated $J_{1}+J_{2}$. Treating $\tilde{I}_{3}, I_{4}$, and $\tilde{I}_{4}$ in a similar fashion, this gives, from (4.10),

$$
\begin{aligned}
& \iint\left\{|u-v| \psi_{t}+\operatorname{sgn}(u-v)[f(u)-f(v)] \psi_{x}\right\} d x d t \\
& \quad \geq\left. 2 \int|u-v| \psi\right|_{t=T} d x-\left.2 \int|u-v| \psi\right|_{t=0} d x-E_{4} .
\end{aligned}
$$

Let $a_{\varepsilon}(x)$ be a nonnegative monotone function such that $a_{\varepsilon}(x)=1$ if $x \geq \varepsilon$, and $a_{\varepsilon}(x)=0$ if $x \leq-\varepsilon$; see Figure 12. Then define $A_{\varepsilon}(x, t)$ by

$$
A_{\varepsilon}(x, t)=1-a_{\varepsilon}(|x|+L t-R+\varepsilon),
$$




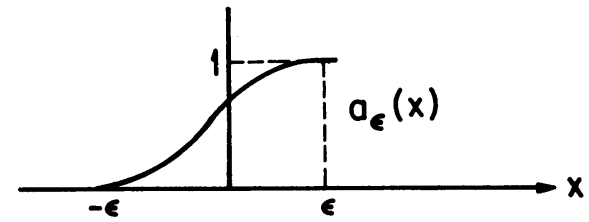

FiguRe 12

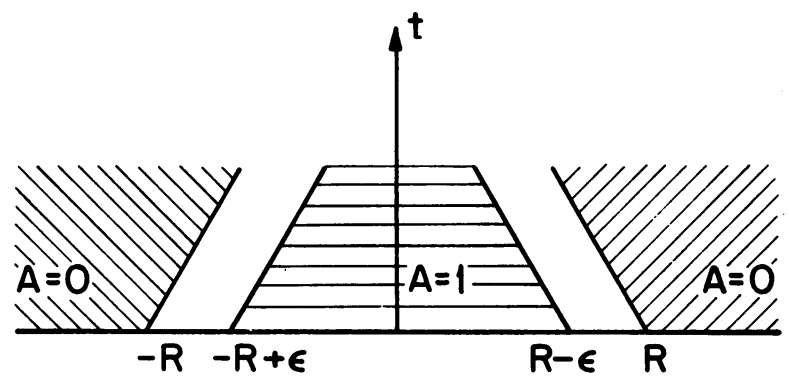

Figure 13

where $R>0$ and $L$ is the Lipschitz constant for $f$ on $|u| \leq\left\|v_{0}\right\|_{\infty}$. Note that if $|x|<R-L t-2 \varepsilon, a_{\varepsilon}(|x|+L t-R+\varepsilon)=0$ so $A_{\varepsilon}(x, t)=1$, and if $|x| \geq$ $R-L t, a_{\varepsilon}(|x|+L t-R+\varepsilon)=1$, so $A_{\varepsilon}(x, t)=0$; see Figure 13. Thus $A_{\varepsilon}$ can serve as $\psi$.

Using this test function in (4.11) gives

$$
\begin{aligned}
\left.2 \int|u-v| A_{\varepsilon}\right|_{t=T-} d x-\left.2 \int|u-v| A_{\varepsilon}\right|_{t=0}-E_{4} \\
\leq \iint\left\{|u-v|\left(A_{\varepsilon}+\frac{\partial A_{\varepsilon}}{\partial t}\right)\right. \\
\left.\quad+\operatorname{sgn}(u-v)[f(u)-f(v)] \frac{\partial A_{\varepsilon}}{\partial x}\right\} d x d t \\
=\iint\left\{|u-v|\left(-L a_{\varepsilon}^{\prime}\right)+\operatorname{sgn}(u-v)[f(u)-f(v)]\left( \pm a_{\varepsilon}^{\prime}\right)\right\} d x d t
\end{aligned}
$$

But

$$
|u-v|\left(-a_{\varepsilon}^{\prime}\right)\left[L \pm \frac{f(u)-f(v)}{u-v}\right] \leq 0
$$

so that

$$
\left.2 \int|u-v| A_{\varepsilon}\right|_{t=T-} d x \leq\left. 2 \int|u-v| A_{\varepsilon}\right|_{t=0}+E_{4},
$$

where $E_{4}$ is defined in (4.10). Thus, from (4.12) we get

$$
\begin{aligned}
& \int_{-R+L t+\varepsilon}^{R-L T-\varepsilon}|u(x, T-)-v(x, T-)| d x \\
& \quad \leq \int_{-R-\varepsilon}^{R+\varepsilon}|u(x, 0)-v(x, 0)| d x+\frac{1}{2} E_{4}
\end{aligned}
$$


where

$$
E_{4}=\left(h^{-2} \Delta t^{1 / 2}|\log \Delta t|+\left(h^{-2} \Delta t+h\right)+\|u-v\|_{1} h\right) O(1),
$$

and the $O(1)$ term includes the derivatives of $\psi$. It follows that

$$
E_{4}=\left(h^{-2} \Delta t^{1 / 2}|\log \Delta t|+h\right) O(1) .
$$

Thus, if we set $h=\Delta t^{1 / 6}$, we get

$$
E_{4}=\left(\Delta t^{1 / 6}|\log \Delta t|+\Delta t^{1 / 6}\right) O(1) .
$$

This yields the following theorem. (Recall that $\Delta t=O(\Delta x)$.)

THEOREM 4.2. Let $v$ be the solution of (1.1), (1.2), and suppose $v(x, 0)$ has finite total variation. If $u$ is a Glimm approximate solution to (1.1), (1.2) ${ }^{4}$ and $X=(a, b)$ is any bounded interval on the line $t=T>0$, there is a constant $C$, depending only on $X, \mathrm{~T} . \mathrm{V} .(v(x, 0))$, and the equidistributed sequence, such that for any $\varepsilon>0$,

$$
\|u-v\|_{L^{1}(X)} \leq C\left[\left\|u_{0}-v_{0}\right\|_{L^{1}(\tilde{X})}+\Delta x^{1 / 6}|\log \Delta x|\right]
$$

where $\tilde{X}=(a-L t-\varepsilon, b+L t+\varepsilon)$, and $L$ is the Lipschitz constant for $f$ on $\left\{u:|u| \leq\left\|v_{0}\right\|_{\infty}\right\}$.

5. Error bounds for some special classes of data. In this section we show that the general error bound (4.14) can be improved in the case that the initial data is piecewise constant.

Throughout this section $v$ denotes the exact solution of (1.1) with initial data $v_{0}$, and $u$ denotes the Glimm approximate solution with initial data $u_{0}$ generated from the particular equidistributed sequences $\left\{a_{n}\right\}$ as described in $\S 1$. In addition, it will be convenient to adopt the notation

$$
h^{p}=\Delta x^{p}|\log \Delta x|=O\left(\Delta t^{p}|\log \Delta x|\right) .
$$

The main result of this section is then the following theorem.

THEOREM 5.1. Assume that

(a) $v_{0}$ is piecewise constant;

(b) $v$ contains no interactions of more than two waves at a time; and

(c) $u_{0}(x)=(1 / 2 \Delta x) \int_{x_{k-1}}^{x_{k+1}} v_{0}(s) d s, \quad x_{k-1}<x<x_{k+1}$.

Then for any $T, X>0$ there is a constant $C$ independent of $\Delta t$ and $\Delta x$ such that, for $0 \leq t \leq T$,

$$
\|u(\cdot, t)-v(\cdot, t)\|_{L^{1}[-X, X]} \leq C h^{1 / 2} .
$$

In addition, if $v$ contains no interactions of shocks with rarefaction waves, then

$$
\|u(\cdot, t)-v(\cdot, t)\|_{L^{1}[-X, X]} \leq C h .
$$

In proving Theorem 5.1 we shall make repeated use of the following two lemmas.

\footnotetext{
${ }^{4}$ Corresponding to any equidistributed sequence satisfying (1.4).
} 
LEMMA 5.2. Suppose there are functions $\alpha(x, t)$ and $\beta(t)$ such that

$$
u(x, t)=v(x+\alpha(x, t), t+\beta(t))
$$

for $0 \leq t \leq T$ and $|x| \leq X$. Then for $0 \leq t \leq T$,

$$
\|u(\cdot, t)-v(\cdot, t)\|_{L^{1}[-X, X]} \leq C\left(\|\alpha\|_{\infty}+\|\beta\|_{\infty}\right) .
$$

ProOF. We have

$$
\begin{aligned}
\int_{-X}^{X}|u(x, t)-v(x, t)| d x \leq & \int_{-X}^{X}|v(x+\alpha, t+\beta)-v(x, t+\beta)| d x \\
& +\int_{-X}^{X}|v(x, t+\beta)-v(x, t)| d x .
\end{aligned}
$$

Interpreting $v_{x}$ as a measure, we can bound the first integral on the right side by

$$
\int_{-X}^{X} \int_{x-\|\alpha\|_{\infty}}^{x+\|\alpha\|_{\infty}}\left|v_{x}(s, t+\beta)\right| d s d x \leq 2\|\alpha\|_{\infty} T V(v(\cdot, t+\beta)) .
$$

Since a similar estimate holds for the second integral in (5.4), (5.3) follows.

LEMMA 5.3. There is a constant $C$ depending only on the equidistributed sequence $\left\{a_{n}\right\}$ such that, for any number s satisfying $|s \Delta t / \Delta x| \leq 1$, the bound

$$
\left|\sum_{m=1}^{n} \operatorname{sgn}\left(s \Delta t-a_{m} \Delta x\right) \Delta x-s t_{n}\right| \leq C(\Delta x \log n)
$$

is valid.

Proof. We have from (1.4) that

$$
\begin{aligned}
\frac{1}{n} \sum_{m=1}^{n} & {\left[\frac{\operatorname{sgn}\left(s \Delta t-a_{m} \Delta x\right)+1}{2}\right] \Delta x } \\
= & \frac{\#\left\{m \in[1, n]: a_{m} \leq s \Delta t / \Delta x\right\}}{n} \Delta x \\
\quad= & \frac{1+s \Delta t / \Delta x}{2} \Delta x+O\left(\frac{\log n}{n}\right) \Delta x .
\end{aligned}
$$

Multiplying by $2 n$ we obtain

$$
\sum_{m=1}^{n} \operatorname{sgn}\left(s \Delta t-a_{m} \Delta x\right) \Delta x+n \Delta x=\left(1+s \frac{\Delta t}{\Delta x}\right) n \Delta x+O(\Delta x \log n),
$$

as required.

Next, we analyze in detail the behavior of the approximate solution $u$ for a number of special cases. The proof of Theorem 5.1 will follow easily from these.

Problem 1. Assume that $v$ consists of two constant states separated by a single shock emanating from $\left(b^{0}, 0\right)$ (Figure 14a) and $u$ consists of the same two states with a discontinuity initially at $\left(z^{0}, 0\right)$ (Figure $14 \mathrm{~b}$ ).

In this case we show that we can choose $\alpha=\alpha(x)$ and $\beta=0$ in Lemma 5.2, and

$$
\|\alpha\|_{\infty} \leq\left|b^{0}-z^{0}\right|+C h
$$




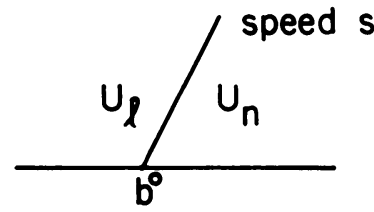

(a) $v(x, t)$

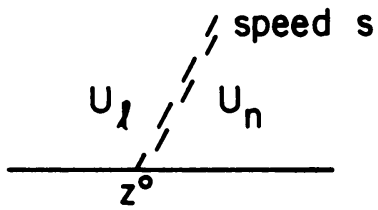

(b) $u(x, t)$

FIGURE 14

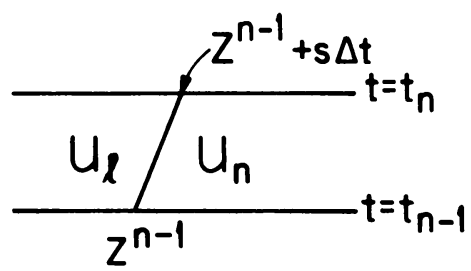

FIGURE 15

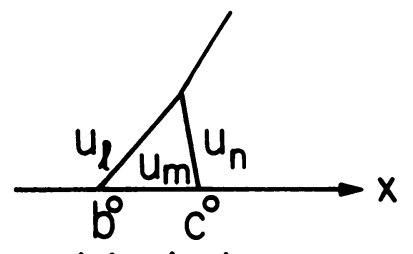

(a) $v(x, t)$

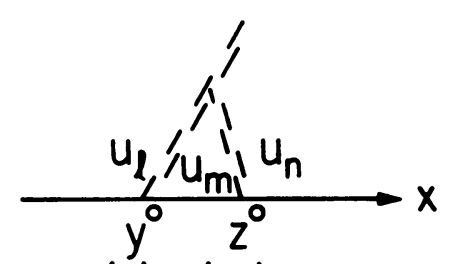

(b) $u(x, t)$

FIGURE 16

PROOF. Let $z^{n}$ be the position of the shock in $u$ at time $t_{n}$. Then from Figure 15 we see that

$$
z^{n}=z^{n-1}+\Delta x \operatorname{sgn}\left(s \Delta t-a_{n} \Delta x\right)
$$

so that, by Lemma 5.3 ,

$$
z^{n}=z^{0}+\sum_{m=1}^{n}\left(s \Delta t-a_{m} \Delta x\right) \Delta x=z^{0}+s t_{n}+O(\Delta x \log n) .
$$

But if $t_{n} \leq T$, then $n=t_{n} / \Delta t \leq T / \Delta t$, so that

$$
z^{n}=z^{0}+s t_{n}+O(h)
$$

On the other hand, the position of the shock in $v$ at time $t_{n}$ is $b^{n}=b^{0}+s t_{n}$. Therefore,

$$
z^{n}-b^{n}=z^{0}-b^{0}=O(h) .
$$

It is now clear that we can choose $\alpha=\alpha(x), \beta=0$, and

$$
\|\alpha\|_{\infty} \leq \max _{n}\left|z^{n}-b^{n}\right| \leq\left|z^{0}-b^{0}\right|+C h .
$$

Problem 2. Here we consider two similar problems, the analysis being nearly the same for both:

A. $v$ and $u$ consist of the interaction of two shocks as shown in Figure 16. 


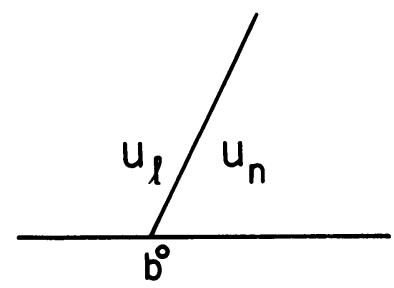

(a) $v(x, t)$

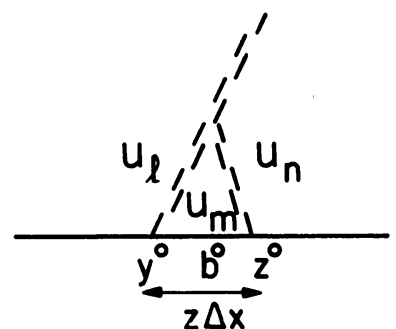

(b) $u(x, t)$

FIGURE 17

B. $v$ is a single shock and, due to initial averaging, $u$ is the interaction of two shocks whose product is the shock in $v$ (Figure 17).

For case B we assume that

$$
u_{m}=\frac{1}{z^{0}-y^{0}} \int_{y^{0}}^{z^{0}} v_{0}(x) d x
$$

and $y^{0}$ and $z^{0}$ are adjacent mesh points. In both $\mathrm{A}$ and $\mathrm{B}, u_{l}>u_{m}>u_{r}$.

For case $\mathrm{A}$ we show that the $\alpha$ and $\beta$ of Lemma 5.3 can be chosen so that

$$
\|\alpha\|_{\infty}+\|\beta\|_{\infty} \leq C\left(\left|b^{0}-y^{0}\right|+\left|c^{0}-z^{0}\right|+h\right) .
$$

For case $\mathrm{B}$ we show that there is a time $t_{\bar{n}}=O(h)$ such that $\|u(\cdot, t)-v(\cdot, t)\|_{L^{1}} \leq C h$ holds for $0 \leq t \leq t_{\bar{n}}$, and, for $t \geq t_{\bar{n}}$, the $\alpha$ and $\beta$ of Lemma 5.3 can be chosen so that $\beta=0, \alpha=\alpha(x)$, and $\|\alpha\|_{\infty} \leq C h$.

PROOF. Let $y^{n}$ and $z^{n}$ be the $x$-coordinates of the two shocks in $u$ at time $t_{n}$. Then, as in Problem 1,

$$
\begin{aligned}
& y^{n}=y^{0}+\sum_{m=1}^{n} \operatorname{sgn}\left(s_{y} \Delta t-a_{m} \Delta x\right) \Delta x, \\
& z^{n}=z^{0}+\sum_{m=1}^{n} \operatorname{sgn}\left(s_{z} \Delta t-a_{m} \Delta x\right) \Delta x,
\end{aligned}
$$

where $s_{y}$ and $s_{z}$ are the shock speeds. Now suppose the two shocks interact at the point $\left(x_{\bar{k}}, t_{\bar{n}}\right)$. Then $y_{\bar{n}}=z_{\bar{n}}=x_{\bar{k}}$, and subtracting in (5.6) and applying Lemma 5.3 , we find that

$$
z^{0}-y^{0}=\left(s_{y}-s_{z}\right) t_{\bar{n}}+O(\Delta x \log \bar{n}) .
$$

We need to obtain an upper bound for $t_{\bar{n}}$. (This will show, in particular, that the interaction in $u$ actually occurs.) First observe that since $f^{\prime \prime}>0,\left|s_{y}-s_{z}\right| \geq$ $C\left|u_{r}-u_{l}\right|$, and this last term is bounded away from zero.

Thus, from (5.7),

$$
t_{\bar{n}}=\left(z^{0}-y^{0}\right) /\left(s_{y}-s_{z}\right)+O\left(\Delta x \log t_{\bar{n}}-\Delta x \log \Delta t\right),
$$

so that

$$
t_{\bar{n}}-C_{1} \Delta x \log t_{\bar{n}} \leq C_{2}\left|z^{0}-y^{0}\right|+C_{3} h .
$$

Hence, $t_{\bar{n}}$ cannot be arbitrarily large. Moreover, in case B we have $z^{0}-y^{0}=2 \Delta x$, so that, when $\Delta x$ is small,

$$
t_{\bar{n}} \leq C_{1} \Delta x \log t_{\bar{n}}+C_{4} h \leq C_{1} h \log t_{\bar{n}} / h+C_{4} h .
$$



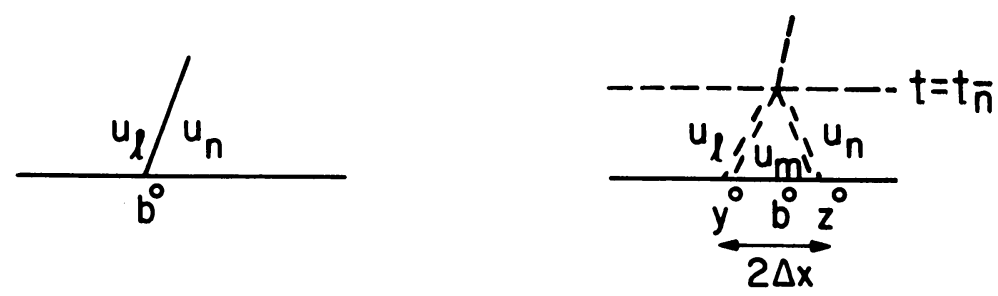

\section{FIGURE 18}

Dividing by $h$, we find that

$$
t_{\bar{n}} / h-C_{1} \log t_{\bar{n}} / h \leq C_{4}
$$

and therefore

$$
t_{\bar{n}} \leq \text { const } h \quad \text { (case B). }
$$

Now consider case $\mathrm{A}$. The point at which the two initial shocks in $v$ interact is $(\bar{x}, \bar{t})$, where

$$
\bar{x}=b_{0}+s_{y} \bar{t} \text { and } \bar{t}=\left(b^{0}-c^{0}\right) /\left(s_{z}-s_{y}\right) .
$$

Therefore, from (5.7) we have

$$
\bar{t}-t_{\bar{n}}=O\left(\left|z^{0}-c^{0}\right|+\left|y^{0}-b^{0}\right|+h\right) .
$$

Next, from (5.8) and Lemma 5.3,

$$
\begin{aligned}
x_{\bar{k}} & =y^{\bar{n}}=y^{0}+\Delta x \sum_{m=1}^{\bar{n}} \operatorname{sgn}\left(s_{y} \Delta t-a_{m} \Delta x\right) \\
& =y^{0}+t_{\bar{n}} s_{y}+O(h) .
\end{aligned}
$$

Therefore, using (5.9), we conclude that

$$
\begin{aligned}
\left|x_{\bar{k}}-\bar{x}\right| & \leq\left|y^{0}-b^{0}\right|+\left|s_{y}\right|\left|t_{\bar{n}}-\bar{t}\right|+O(h) \\
& \leq C\left(\left|y^{0}-b^{0}\right|+\left|z^{0}-c^{0}\right|+h\right) .
\end{aligned}
$$

This estimate, together with (5.9), completes the analysis for case A.

We now consider case B. First note that the estimate $\|u(\cdot, t)-v(\cdot, t)\|_{L^{1}} \leq C h$ is obvious for $t \in\left[0, t_{\bar{n}}\right]$ (see Figure 18).

At $t=t_{\bar{n}}, u$ has as "data" a single shock emanating from the point $\left(x_{\bar{k}}, t_{\bar{n}}\right)$, where by $(5.10)$,

$$
x_{\bar{k}}=y^{0}+t_{\bar{n}} s_{y}+O(h) .
$$

On the other hand, this same shock is located in $v$ at $\left(x, t_{\bar{n}}\right)$, where $x=b^{0}+s t_{\bar{n}}$. Thus

$$
\left|x_{\bar{k}}-x\right| \leq\left|y^{0}-b^{0}\right|+\left|s_{y}-s\right| t_{\bar{n}}+O(h) \leq O(h)
$$

by (5.8). We can now apply the result of Problem 1 with $t=t_{\bar{n}}$ as the new initial time to define $\alpha$ and $\beta$ as required.

Problem 3. Here the exact solution $v$ is the rarefaction wave shown in Figure 19a.

We make the assumption that the characteristic speeds in the interior of this wave are strictly increasing, so that each state $w \in\left(u_{l}, u_{r}\right)$ is achieved at a unique point $b(w)$ by $v_{0}$; that is, $v_{0}(b(w))=w$. 

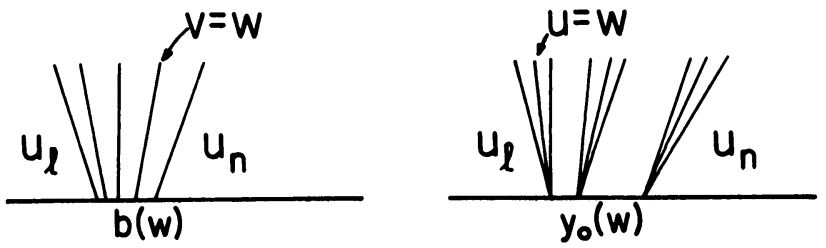

FIGURE 19

As for $u$, we assume that there are a finite number of adjacent mesh points which are centers of subwaves in $\mathbf{R} \times[0, \Delta t]$ (Figure 19b), and that the union of these subwaves is the wave in $v$. If $w$ is a state achieved by $u$ in the interior of one of these subwaves, we denote by $y^{0}(w)$ the mesh point at which that particular subwave is achieved.

We show that, under these assumptions, the $\alpha$ and $\beta$ of Lemma 5.3 can be defined so that $\alpha=\alpha(x), \beta=0$, and

$$
\|\alpha\|_{\infty} \leq C\left[\sup _{w}\left|b(w)-y^{0}(w)\right|+h\right]
$$

where the sup is taken over all $w$ for which $y^{0}(w)$ is defined. In addition, we also show that

$$
\left|u_{k+1}^{n}-u_{k-1}^{n}\right| \leq C h / t_{n} .
$$

This estimate is required for the analysis in Problem 4.

PROOF. We partition each of the waves in $u$ so that no further partitioning occurs before a given time $T$ (recall §2B). Let $w$ be any state achieved by $u$ in the interior of one of these subwaves, and let $y^{n}$ be the $x$-coordinate of the center of the subwave in $u$ at time $t_{n}$. Then, as in Problems 1 and 2,

$$
\begin{aligned}
y^{n} & =y^{0}(w)+\sum_{m=1}^{n} \operatorname{sgn}\left(f^{\prime}(w) \Delta t-a_{m} \Delta x\right) \Delta x \\
& =y^{0}(w)+f^{\prime}(w) t_{n}+O(h) .
\end{aligned}
$$

On the other hand, the exact solution $v$ achieves the value $w$ at the point $\left(x, t_{n}\right)$, where $x=b(w)+f^{\prime}(w) t_{n}$. Subtracting, we thus have

$$
\left|y^{n}-x\right| \leq \sup _{w}\left|y_{0}(w)-b(w)\right|+C h .
$$

It now follows easily that $\alpha$ and $\beta$ can be defined as claimed.

To prove (5.12) we simply note that, for $\delta$ small, by (5.13),

$$
x_{k}=y^{0}\left(u_{k-1}^{n}+\delta\right)+f^{\prime}\left(y^{0}\left(u_{k-1}^{n}+\delta\right)\right) t_{n}+O(h),
$$

and

$$
x_{k}=y^{0}\left(u_{k+1}^{n}-\delta\right)+f^{\prime}\left(y^{0}\left(u_{k+1}^{n}+\delta\right)\right) t_{n}+O(h) .
$$

Since $y^{0}\left(u_{k-1}^{n}+\delta\right)=y^{0}\left(u_{k+1}^{n}+\delta\right)$, we obtain, upon subtracting and letting $\delta \rightarrow 0$,

$$
f^{\prime}\left(u_{k+1}^{n}\right)-f^{\prime}\left(u_{k-1}^{n}\right)=O\left(h / t_{n}\right) .
$$

Since $f^{\prime \prime}>0,(5.12)$ follows. 


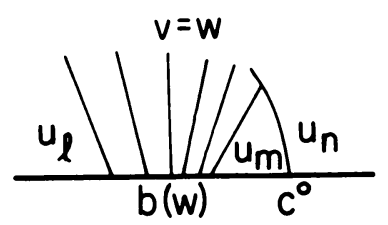

(a) $v(x, t)$

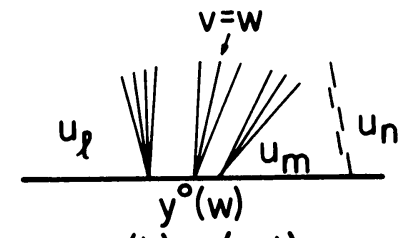

(b) $u(x, t)$

FIGURE 20

Problem 4. Here the exact solution $v$ consists of a shock wave interacting with a rarefaction wave. As before, we assume that the characteristic speeds are strictly increasing in the interior of the rarefaction wave. The functions $b(w)$ and $y^{0}(w)$ are then defined just as in Problem 3. (See Figure 20.)

We show that, for this problem, the $\alpha$ and $\beta$ of Lemma 5.2 can be defined in such a way that

$$
\|a\|_{\infty}+\|\beta\|_{\infty} \leq C\left[\sup _{w}\left|b(w)-y^{0}(w)\right|+\left|c^{0}-z^{0}\right|+h\right] .
$$

PROOF. Let $s_{u}^{n}$ be the speed of the shock in the approximate solution $u$ for $t_{n}<t<t_{n+1}$. We claim that

$$
s_{u}^{n+1}-s_{u}^{n}=O(h) .
$$

To see this, note that the first interaction in $u$ of the shock with a subwave of the rarefaction wave occurs at a time $t_{\bar{n}}$ bounded away from $0 ;(5.14)$ is then obvious for $t_{n}<t_{\bar{n}}$. Now for $t_{n} \geq t_{\bar{n}}, s_{u}^{n+1}-s_{u}^{n}=O\left(u_{k+1}^{n}-u_{k}^{n}\right)$ if the shock at $\left(x_{k+2}, t_{n}\right)$ interacts with a subwave at $\left(x_{k}, t_{n}\right) ;(5.14)$ then follows from (5.12).

Now let $z^{n}$ be the $x$-coordinate of the shock in $u$ at time $t_{n}$. Then, as before,

$$
z^{n}=z^{0}+\sum_{m=1}^{n} \operatorname{sgn}\left(s_{u}^{m} \Delta t-a_{m} \Delta x\right) \Delta x .
$$

At this point we require the following refinement of Lemma 5.3.

LEMMA 5.4. Assume that $\left\{g^{m}\right\}_{m=1}^{\infty}$ is a sequence of real numbers satisfying $\left|g^{m}\right| \leq 1$ and $\left|g^{m+1}-g^{m}\right| \leq C h$. If $K$ is a positive integer with $K=O\left(\Delta t^{-1 / 2}\right)$, then

$$
\frac{1}{n} \sum_{m=1}^{n} \operatorname{sgn}\left(g^{m}-a_{m}\right)=\frac{1}{t_{n}} \sum_{j=1}^{\left[t_{n} / K \Delta t\right]} g_{j K} \cdot K \Delta t+O\left(h^{1 / 2}\right) .
$$

The proof of Lemma 5.4 is given in the Appendix.

Now choosing $K=O\left(\Delta t^{-1 / 2}\right)$ throughout, we therefore have from (5.15) that

$$
\begin{aligned}
z^{n} & =z^{0}+n \Delta x\left[\frac{1}{n} \sum_{1}^{n} \operatorname{sgn}\left(s_{u}^{m} \frac{\Delta t}{\Delta x}-a_{m}\right)\right] \\
& =z^{0}+n \Delta x\left[\frac{1}{t_{n}} \sum_{j=1}^{\left[t_{n} / K \Delta t\right]} s_{u}^{j K} \frac{\Delta t}{\Delta x} \cdot K \Delta t\right]+O\left(h^{1 / 2}\right) \\
& =z^{0}+\sum_{j=1}^{\left[t_{n} / K \Delta t\right]} s_{u}^{j K} \cdot K \Delta t+O\left(h^{1 / 2}\right) .
\end{aligned}
$$


We now extend the sequences $z^{n}$ and $s_{u}^{n}$ by piecewise linear interpolation to obtain functions $z(t)$ and $s_{u}(t)$ defined for all $t \geq 0$. From (5.14) and (5.15) we then have for $t, t^{\prime} \geq 0$,

$$
\left\{\begin{array}{l}
\left|s_{u}(t)-s_{u}\left(t^{\prime}\right)\right| \leq C\left|t-t^{\prime}\right||\log \Delta t| \\
\left|z(t)-z\left(t^{\prime}\right)\right| \leq C\left|t-t^{\prime}\right|
\end{array}\right.
$$

The sum in (5.16) may thus be rewritten as

$$
\begin{aligned}
\sum_{j=1}^{\left[t_{n} / K \Delta t\right]} s_{u}^{j K} \cdot K \Delta t & =\sum_{j=1}^{\left[t_{n} / K \Delta t\right]}\left[\int_{(j-1) K \Delta t}^{j K \Delta t} s_{u}(t) d t+O\left(K^{2} \Delta t^{2}|\log t|\right)\right] \\
& =\int_{0}^{t_{n}} s_{u}(t) d t+O(K \Delta t|\log \Delta t|) \\
& =\int_{0}^{t_{n}} s_{u}(t) d t+O\left(h^{1 / 2}\right) .
\end{aligned}
$$

We therefore have from (5.16) that

$$
z(t)=z(0)+\int_{0}^{t} s_{u}(t) d t+O\left(h^{1 / 2}\right)
$$

holds for $t=t_{n}$. (5.18) then holds for all $t \geq 0$ by (5.17).

Now, if $c(t)$ and $s_{v}(t)$ are the $x$-coordinate and speed of the shock in $v$ at time $t$, respectively, then

$$
c(t)=c(0)+\int_{0}^{t} s_{v}(t) d t
$$

Subtracting from (5.18), we therefore have

$$
z(t)-c(t)=z(0)-c(0)+\int_{0}^{t}\left[s_{u}(t)-s_{v}(t)\right] d t+O\left(h^{1 / 2}\right)
$$

We show below that

$$
\left|s_{u}(t)-s_{v}(t)\right| \leq\left[\sup _{w}\left|b(w)-y^{0}(w)\right|+h+|z(t)-c(t)|\right] .
$$

Granting this for the moment, we may substitute (5.20) into (5.19) and apply Gronwall's inequality to obtain

$$
|z(t)-c(t)| \leq C\left[\left|z^{0}-c^{0}\right|+\sup _{w}\left|b(w)-y^{0}(w)\right|+h\right] .
$$

The assertions about $\alpha$ and $\beta$ now follow easily.

To prove (5.20) we let $\tilde{v}$ be the solution of (1.1) with initial data

$$
\tilde{v}(x, 0)= \begin{cases}v(x, 0), & x<c^{0} \\ u_{m}, & x \geq c^{0}\end{cases}
$$

and $\tilde{u}$ the approximate solution with data

$$
\tilde{u}(x, 0)= \begin{cases}u(x, 0), & x<z^{0} \\ u_{m}, & x \geq z^{0}\end{cases}
$$




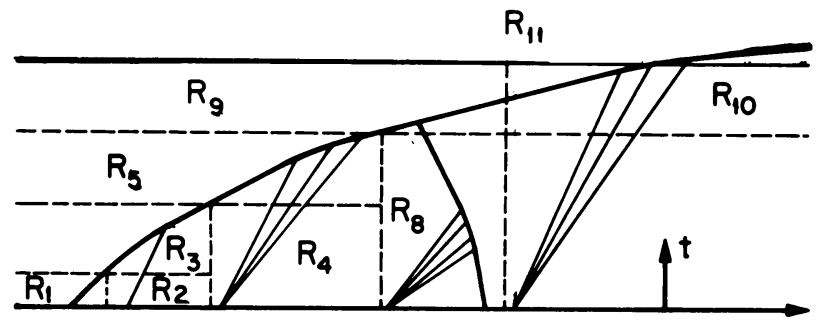

FIGURE 21

Thus $\tilde{v}$ agrees with $v$ for $x<c(t)$, and $\tilde{u}_{k}^{n}$ agrees with $u_{k}^{n}$ for $x_{k}<z^{n}$. Now fix a time $t_{n}$ and let $\bar{v}=v\left(c\left(t_{n}\right)-, t_{n}\right)$ and $\bar{u}=u\left(z\left(t_{n}\right)-, t_{n}\right)$. The Rankine-Hugoniot relation then implies that

$$
\left|s_{u}\left(t_{n}\right)-s_{v}\left(t_{n}\right)\right| \leq C|\bar{u}-\bar{v}| .
$$

However, $\tilde{v}\left(\cdot, t_{n}\right)$ achieves the value $\bar{u}$ at some point $\bar{x}$, so by Lipschitz continuity (5.12),

$$
\begin{aligned}
\left|s_{u}\left(t_{n}\right)-s_{v}\left(t_{n}\right)\right| & \leq C\left|\tilde{v}\left(\bar{x}, t_{n}\right)-\tilde{v}\left(c\left(t_{n}\right), t_{n}\right)\right| \\
& \leq C\left(\left|\bar{x}-z\left(t_{n}\right)\right|+\left|z\left(t_{n}\right)-c\left(t_{n}\right)\right|\right) .
\end{aligned}
$$

On the other hand, since $\tilde{u}\left(z\left(t_{n}\right), t_{n}\right)=\bar{u}=\tilde{v}\left(\bar{x}, t_{n}\right),\left|z\left(t_{n}\right)-\bar{x}\right|$ is bounded by $\|\alpha\|_{\infty}$, where $\alpha$ is as in Problem 3. That is,

$$
\left|z\left(t_{n}\right)-\bar{x}\right| \leq C\left[h+\sup _{w}\left|b(w)-y^{0}(w)\right|\right] .
$$

If we substitute this into (5.21), we obtain (5.20) for $t=t_{n}$. (5.20) then holds for all $t$ in view of (5.19).

ProOF OF THEOREM 5.1. We subdivide the upper half-plane $\{t \geq 0\}$ into nonoverlapping rectangles $R_{i}$ whose bottom and top boundaries are intervals with the lines $t=a_{i}$ and $t=b_{i}$, and in such a way that in each $R_{i}$ the exact solution $v$ consists either of a single wave entering $R_{i}$ at the bottom and leaving $R_{i}$ at the top, or of two waves entering $R_{i}$ at the bottom with the product of their interaction leaving $R_{i}$ at the top (see Figure 21). (By the "bottom" of $R_{i}$ we mean the interior of $\partial R_{i} \cap\left\{t=a_{i}\right\}$; similarly for "top".) If $a_{i}=0$ for a particular $R_{i}$, then our hypotheses show that the results of either problem 2B or Problem 3 become applicable, so that the error bound (5.2) holds in $R_{i}$. Moreover, the existence of $\alpha$ and $\beta$ for $(x, t) \in \partial R_{i} \cap\left\{t=b_{i}\right\}$ establishes the hypotheses of the appropriate problem 1-4 for regions $R_{j}$, whose bottoms intersect the top of $R_{i}$, with errors in the positions of the "initial" waves (i.e., at $t=b_{i}=a_{j}$ ) bounded by $\|\alpha\|_{\infty}+\|\beta\|_{\infty}=O(h)$. Proceeding by induction on $i$ towards increasing time, we obtain $\alpha$ and $\beta$ as in Lemma 5.2, defined in all $R_{i}$ (with the possible exception of those $R_{i}$ to which problem $2 \mathrm{~B}$ applies) and such that $\|\alpha\|_{\infty}+\|\beta\|_{\infty}$ is bounded by $O\left(h^{1 / 2}\right)(O(h)$ when no shock-rarefaction interactions occur). Estimates (5.2) and (5.3) then follow from Lemma 5.3 and Problem 2B.

Appendix. We prove the following lemma which was used in $\S 5$. Recall that $h^{p}=\Delta x^{p}|\log \Delta x|=O\left(\Delta t^{p}|\log \Delta t|\right)$. 
LEMMA 5.4. Assume that $\left\{g^{m}\right\}_{m=1}^{\infty}$ is a sequence of real numbers satisfying $\left|g^{m}\right| \leq 1$ and $\left|g^{m+1}-g^{m}\right| \leq C h$. If $K$ is a positive integer with $K=O\left(\Delta t^{-1 / 2}\right)$, then

$$
\frac{1}{n} \sum_{m=1}^{n} \operatorname{sgn}\left(g^{m}-a_{m}\right)=\frac{1}{t_{n}} \sum_{j=1}^{\left[t_{n} / K \Delta t\right]} g_{j K} \cdot K \Delta t+O\left(h^{1 / 2}\right),
$$

for any equidistributed sequence $\left\{a_{m}\right\} \subset(-1,1)$ which satisfies (1.4).

PROOF. Let $M=\left[t_{n} / K \Delta t\right]=[n / K]$, and consider the following partition of the integers $\{1,2, \ldots, n\}$ :

$$
\begin{gathered}
A_{j}=\{(j-1) K+1,(j-1) K+2, \ldots, j K\}, \quad j=1,2, \ldots, M, \\
A_{M+1}=\{M K+1, M K+2, \ldots, n\} .
\end{gathered}
$$

Then we can write

$$
\begin{aligned}
& \frac{1}{n} \sum_{m=1}^{n} \operatorname{sgn}\left(g^{m}-a_{m}\right) \\
&=\frac{1}{n} \sum_{j=1}^{M+1} \sum_{m \in A_{j}} \operatorname{sgn}\left(g^{m}-a_{m}\right) \\
&=\frac{1}{n}\left\{\sum_{j=1}^{M} \sum_{m \in A_{j}} \operatorname{sgn}\left(g^{j K}-a_{m}\right)\right. \\
& \quad+\sum_{j=1}^{M} \sum_{m \in A_{j}}\left[\operatorname{sgn}\left(g^{m}-a_{m}\right)-\operatorname{sgn}\left(g^{j K}-a_{m}\right)\right] \\
&\left.+\sum_{m \in A_{M+1}} \operatorname{sgn}\left(g^{m}-a_{m}\right)\right\} .
\end{aligned}
$$

Now

$$
\begin{aligned}
\sum_{m \in A_{j}} \operatorname{sgn}\left(g^{j K}-a_{m}\right) & =\frac{1}{\Delta x} \sum_{m \in A_{j}} \operatorname{sgn}\left[g^{j K} \frac{\Delta x}{\Delta t} \Delta t-a_{m} \Delta x\right] \Delta x \\
& =\frac{1}{\Delta x}\left\{g^{j K} \frac{\Delta x}{\Delta t} K \Delta t+O(\Delta x \log K)\right\},
\end{aligned}
$$

where we have used Lemma 5.3. Also, if we define the interval

$$
I_{j}=\left[\min _{m \in A_{j}} g^{m}, \max _{m \in A_{j}} g^{m}\right],
$$

then $\left|I_{j}\right|=O(h K)=O\left(h^{1 / 2}\right)$, and for $1 \leq j \leq M$,

$$
\left|\sum_{m \in A_{j}}\left[\operatorname{sgn}\left(g^{m}-a_{m}\right)-\operatorname{sgn}\left(g^{j K}-a_{m}\right)\right]\right| \leq \#\left\{m \in A_{j}: a_{m} \in I_{j}\right\} .
$$


Since we obviously have $\sum_{m \in A_{M+1}} \operatorname{sgn}\left(g^{m}-a_{m}\right)=O(K)$, it follows from (1.4) that

$$
\begin{aligned}
& \frac{1}{n} \sum_{m=1}^{n} \operatorname{sgn}\left(g^{m}-a_{m}\right)=\frac{1}{n}\left\{\frac{1}{\Delta t} \sum_{j=1}^{M}\left[g^{j K} K \Delta t+O(\Delta x \log K)\right]\right. \\
& \left.\quad+\sum_{j=1}^{M} \#\left\{m \in A_{j}: a_{m} \in I_{j}\right\}+O(K)\right\} \\
& =\frac{1}{t_{n}} \sum_{j=1}^{M} g^{j K} K \Delta t+O\left[\frac{M \log K}{n}+\frac{M K}{n}\left(h^{1 / 2}+\frac{\log K}{K}\right)+\frac{K}{n}\right] \\
& =\frac{1}{t_{n}} \sum_{j=1}^{M} g^{j K} K \Delta t+O\left(h^{1 / 2}\right),
\end{aligned}
$$

because $M K / n \leq 1, K=O\left(\Delta t^{-1 / 2}\right)$, and $1 / n=O(\Delta t)$.

\section{REFERENCES}

[G] J. Glimm, Solutions in the large for nonlinear hyperbolic systems of equations, Comm. Pure Appl. Math. 18 (1965), 95-105.

[GL] J. Glimm and P. Lax, Decay of solutions of systems of non-linear hyperbolic conservation laws, Mem. Amer. Math. Soc. No. 101 (1970).

[K] S. N. Krushkov, First-order quasilinear equations in several space variables, Math. USSR Sb. 10 (1970), 217-273.

[Ku N] L. Kuipers and H. Niederreiter, Uniform distribution of sequences, Wiley, New York, 1974.

[Ku] N. N. Kuznetsov, On stable methods for solving first order partial differential equations in the class of discontinuous functions, Topics in Numerical Analysis II (Proc. Roy. Irish Acad. Conf. on Numerical Analysis, 1976) (J. J. H. Miller, ed.), Academic Press, New York, pp. 183-197.

[L] T. P. Liu, The deterministic version of the Glimm scheme, Comm. Math. Phys. 57 (1977), 135148.

[S] J. Smoller, Shock waves and reaction-diffusion equations, Springer-Verlag, New York, 1983.

DEPARTMENT OF MATHEMATICS, INDIANA UNIVERSITY, BLOOMINGTON, INDIANA 47401 48109

Department of MAthematics, University of Michigan, ANN ARbor, Michigan 